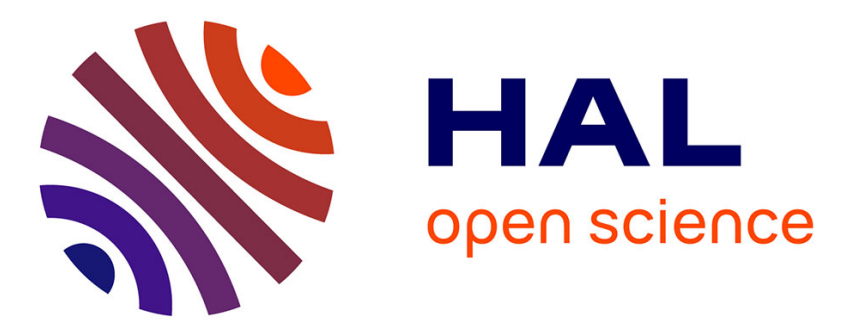

\title{
A new body-fitted immersed volume method for the modeling of ductile fracture at the microscale: Analysis of void clusters and stress state effects on coalescence
} Modesar Shakoor, Marc Bernacki, Pierre-Olivier Bouchard

\section{- To cite this version:}

Modesar Shakoor, Marc Bernacki, Pierre-Olivier Bouchard. A new body-fitted immersed volume method for the modeling of ductile fracture at the microscale: Analysis of void clusters and stress state effects on coalescence. Engineering Fracture Mechanics, 2015, 147, pp.398-417. 10.1016/j.engfracmech.2015.06.057 . hal-01181257

HAL Id: hal-01181257

https://hal-mines-paristech.archives-ouvertes.fr/hal-01181257

Submitted on 29 Apr 2019

HAL is a multi-disciplinary open access archive for the deposit and dissemination of scientific research documents, whether they are published or not. The documents may come from teaching and research institutions in France or abroad, or from public or private research centers.
L'archive ouverte pluridisciplinaire HAL, est destinée au dépôt et à la diffusion de documents scientifiques de niveau recherche, publiés ou non, émanant des établissements d'enseignement et de recherche français ou étrangers, des laboratoires publics ou privés. 


\title{
A new body-fitted immersed volume method for the modeling of ductile fracture at the microscale: analysis of void clusters and stress state effects on coalescence
}

\author{
Modesar Shakoor ${ }^{\mathrm{a}, *}$, Marc Bernacki ${ }^{\mathrm{a}}$, Pierre-Olivier Bouchard ${ }^{\mathrm{a}}$ \\ ${ }^{a}$ Mines-ParisTech, PSL-Research University, CEMEF - Centre de mise en forme des matériaux, CNRS UMR 7635, CS \\ 10207 rue Claude Daunesse 06904 Sophia Antipolis Cedex, France.
}

\begin{abstract}
In this work, a new finite element framework is developed and applied to the study and modeling of ductile fracture mechanisms at the microscale. More particularly, a body-fitted meshing and remeshing methodology is introduced and applications to void coalescence are investigated. Though most studies focus on periodic arrangements of voids, it was proven in experiments as in simulations that random void clusters have a major influence on void growth and coalescence. With the method proposed in this paper, various void arrangements can be addressed and their effect on void growth and coalescence can be studied at large plastic strain and various stress states.
\end{abstract}

Keywords: ductile fracture; finite element modeling; coalescence; void clusters

\section{Introduction}

Ductile fracture raises many challenges in experimentation as in modeling and simulation. In the industry, predicting ductile fracture is usually based on simulations using macroscopic ductile damage models, which are calibrated using experiments where measures are also taken at the macroscale. Example of model which covers such use is the Lemaitre damage model, for which a detailed implementation from experimental calibration to numerical simulation can be found in [1]. In [2], this model was applied to several multi-pass industrial processes and compared to the Gurson-Tvergaard-Needleman microscopic ductile damage model $[3,4]$. For the studied materials and processes, the results showed the superiority of the GTN model, which was the only one to assess individually each of the three mechanisms of ductile damage at the microscale: void nucleation, growth and coalescence. While the first microscopic damage models were designed only for specific conditions, particularly regarding loading path, multiple extensions were proposed in the literature $[5,6,7,8,9,10,11,12,13]$. The main aspect of these extensions is to model more accurately the considered materials and their microstructures. The methodology to reach such objectives is based on Finite Element (FE) analysis: an ideal microstructure composed of a periodic arrangement of spherical or elliptical voids is placed in an infinite matrix. Inclusions can be considered instead of voids to study void nucleation. Applying various loadings and also varying the geometry of the microstructure enables to postulate void evolution laws. These laws are directly used as input to microscopic damage models, such as the GTN model. Due to important computational costs, these studies mostly address a limited number of voids, and the simulation is stopped at the onset of void nucleation or coalescence. Hence, no study of the impact of these two events on material behavior can be performed.

In [14], a FE framework was proposed to simulate void growth at large plastic strain, and an anisotropic mesh adaptation strategy was used to reduce computational costs. This framework was then extended with

\footnotetext{
* Corresponding author.

Email address: modesar.shakoor@mines-paristech.fr (Modesar Shakoor)
} 
void nucleation models and simulations could be pursued after the onset of void nucleation [15]. In this immersed volume framework, a single mesh was used to model the whole heterogeneous material, and the interfaces between matrix, voids and inclusions were not carried by faces of this mesh but by elements for which the behavior was ruled by mixture laws. The main reason for such a choice relied on the complexity of meshing and remeshing interfaces using the mesh adaptation tool presented in [16, 17].

In the first part of the present paper, a new mesh adaptation tool is proposed to solve this issue. An accurate description of matrix/void and matrix/inclusion interfaces is obviously important to compute more accurately the local mechanical variables that play a major role in the prediction of void nucleation and coalescence. As a first utilization of this new remeshing methodology, the second part of the present paper focuses on the simulation of void coalescence. At the microscale, which is addressed here, the prediction of void coalescence is based on local mechanical states. Then, a numerical technique is presented to model void coalescence and to pursue the simulation after its occurrence. In a third and last part, numerical validation of this framework is addressed before studying the influence of void clusters and stress state on ductility for complex void configurations, which can be considered thanks to the proposed remeshing methodology.

\section{Numerical framework}

In a classical immersed volume framework, the simulation is performed using only one mesh, on which the mechanical problem is solved once for all phases. Inside this mesh, the different phases are hence to be described. In [14], the Level-Set (LS) method was used to represent void/matrix interfaces. The LS function $\psi$ of the void/matrix interface $\Gamma$ was defined by:

$$
\forall t,\left\{\begin{aligned}
\psi(x, t) & = \pm d(x, \Gamma(t)), x \in \Omega, \\
\Gamma & =\{x \in \Omega, \psi(x, t)=0\},
\end{aligned}\right.
$$

where $d(.,$.$) is the Euclidean distance, with a positive sign inside the void phase, and a negative one in the$ matrix. As a P1 formulation was used, this signed distance function was computed at mesh nodes at the initial state, and then the evolution of the interface was taken into account by Lagrangian displacement of the nodes during the computation. Other modifications of the interface such as the consequences of void nucleation were operated directly on the LS function [15].

The LS method is classically used to model fluid-structure interactions. However, in these cases the deformation of the solid phase is usually neglected as the focus is made on the flow or on thermal exchanges. Hence, the authors impose the nullity of the solid's deformation rate by defining its behavior as a fluid with penalized viscosity $[18,19,20]$. In these works, the interfaces are said to be implicit, as they are not carried by the mesh but only by the LS function. Consequently, some elements are crossed by the LS function and their behavior is defined by a mixture law taking into account both fluid and solid behaviors. As both fluid and solid phases are modeled by a fluid behavior, this mixture law simply consists in mixing the viscosities in the stationary case. When the deformation of the solid is of interest, this principle can also be applied for nonlinear behaviors such as visco-plasticity. When mechanical properties other than viscosity come into play, such as in visco-elasticity [21] or crystal plasticity [22], homogenizing both fluid and solid behavior becomes more complex. 


\section{mixture}

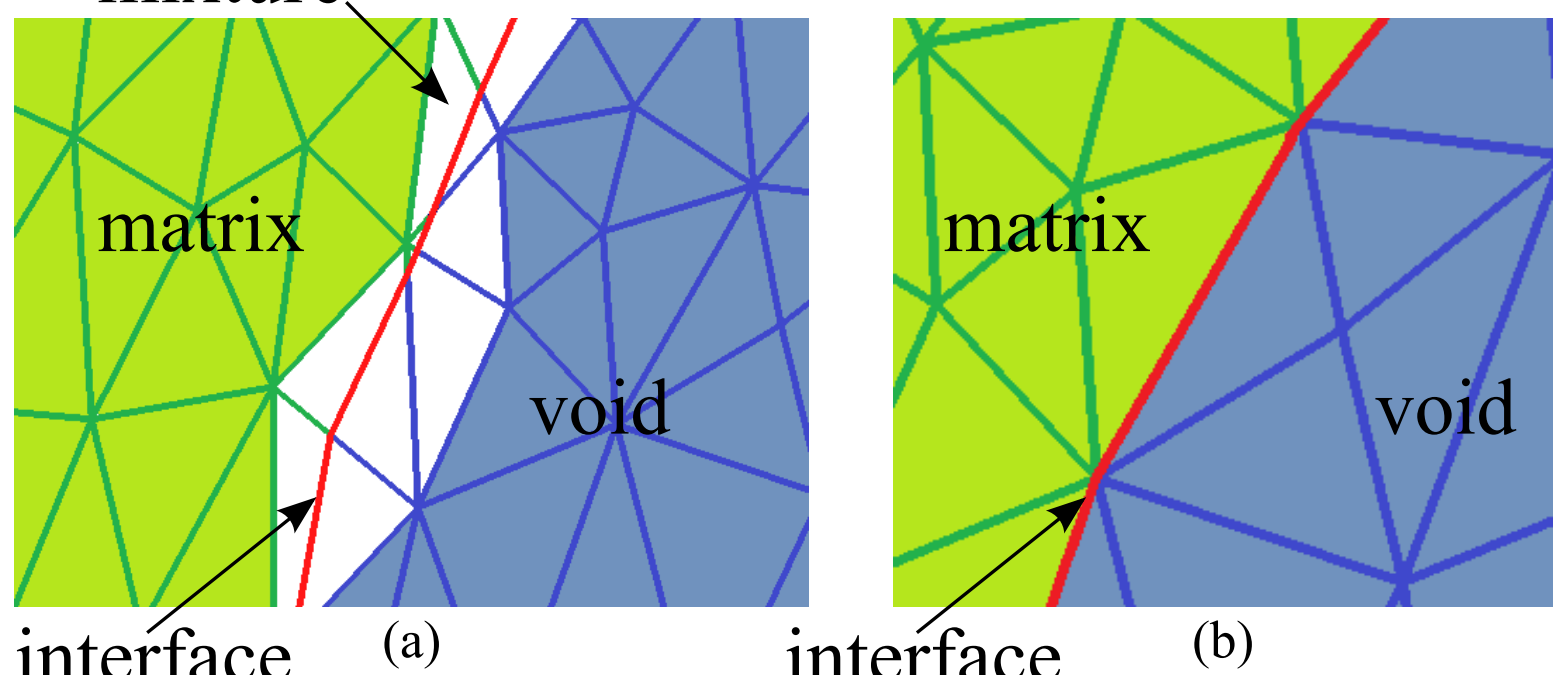

Figure 1: Immersed volume mesh with an implicit interface in a P1 formulation (a), and an explicit (body-fitted) interface (b).

A way to avoid such difficulties would be to avoid having elements crossed by the interface, and hence dealing with an explicit interface (i.e. carried by the mesh), as illustrated in Fig. 1. While the above-mentioned approaches were focused on the mathematical formulation, meshing the interfaces and maintaining a good mesh quality around them during simulation requires important algorithmic developments. In [23, 24, 25], a complete mesh adaptation framework was built to enable fluid-structure simulations with explicit interfaces. Though some results were obtained with great deformations for the solid phase, these deformations remained in the frame of incompressibility, which ensures that refinement or coarsening of surface meshes is not necessary. In the present work, void growth is studied at large plastic strain, using an updated Lagrangian formulation. Voids may grow more than ten times their initial size and importantly distort the mesh, hence surface meshes will certainly need to be remeshed. That is the reason why a new mesh adaptation framework was developed. The parallel $\mathrm{C}++$ library CimLib, which is used for the present developments, has already been used by multiple authors for simulations based on the LS method [14, 15, 18, 19, 20, 21, 22, 26]. For remeshing operations, the external C (re)mesher MTC was used [16]. In the following sections, a new mesh adaptation tool which enables simulations with internal interfaces is presented, and the algorithms that were added to the library to achieve better robustness are detailed.

\subsection{Body-fitted (re)meshing}

MTC is a P1 automatic remesher based on elements topology improvement that was developed for Lagrangian simulations under large strains. This tool was extended to anisotropic mesh adaptation, for which it was extensively used $[14,15,18,19,20,21,22,26]$.

In the present work, four operations were to be added to this software, which are namely fitting, locking, refining and coarsening of an interface described by a LS function. Due to the fact that MTC was initially developed many years ago, it was chosen to produce a fresh implementation, containing the anisotropic mesh adaptation operations already present in MTC, but also the four new operations. This new mesh adaptation tool, named Fitz, was programmed in $\mathrm{C}$ in order to achieve good performance with a code as light and simple as possible. A particular attention was given to data structures and sorting algorithms during its conception. Such issues have been repetitively discussed in the literature for the development of new mesh adaptation tools [24, 27], and will not be detailed here.

Before describing in details the new mesh adaptation operations included in Fitz, some notations and principles have to be introduced. Here, only the remeshing problem will be addressed. An initial mesh is thus considered, which can be defined by a set of nodes $\mathcal{N}_{h}$ and simplexes $\mathcal{T}_{h}$ (triangles in 2D and tetrahedrons 
in $3 \mathrm{D}$ ). Noting $d$ the dimension (2 or 3 ), a simplex of the mesh is given by a set of $d+1$ nodes belonging to $\mathcal{N}_{h}$. The following sets have to be defined:

$$
\begin{aligned}
& \forall n \in \mathcal{N}_{h}, \mathcal{T}(n)=\left\{T \in \mathcal{T}_{h} / n \in T\right\} \\
& \mathcal{N}(\mathcal{T})=\left\{n^{\prime} \in \mathcal{N}_{h} / \exists T \in \mathcal{T}, n^{\prime} \in T\right\}
\end{aligned}
$$

In other words, $\mathcal{T}(n)$ is the set of all simplexes that contain a given node $n$, and the set $\mathcal{N}(\mathcal{T}(n))$ is defined by all the nodes of these simplexes. In the following, the set $\mathcal{T}(n)$ is named neighborhood of $n$. In the same way, the neighborhood of a face can be defined. A face is given by any set of $d$ nodes of the same simplex. The set of the faces of the mesh is noted $F_{h}$, and the neighborhood of a face $F$, noted $\mathcal{T}(F)$, is defined as:

$$
\mathcal{T}(F)=\left\{T \in \mathcal{T}_{h} / \forall n \in T, \forall n^{\prime} \in F, n \in \mathcal{N}\left(\mathcal{T}\left(n^{\prime}\right)\right)\right\}
$$

To drive mesh adaptation, simplex quality has to be defined. Here, the following definition is chosen:

$$
\forall T \in \mathcal{T}_{h}, Q(T)=\min \left(c_{0} \frac{|T|_{M}}{h_{M}^{d}}, h_{M}^{d}, \frac{1}{h_{M}^{d}}\right)
$$

where:

- $c_{0}$ is a normalization factor so that a regular simplex would have a quality of $1, c_{0}=\frac{d !}{\sqrt{d+1}} 2^{d / 2}$,

- $|T|$ is the volume of $T$ and $|T|_{M}=|T| \sqrt{\operatorname{det}(M(T))}$,

- $h_{M}$ is the average edge length $h_{M}=\sqrt{\left(\frac{1}{c_{1}} \sum_{(i, j) \in \mathcal{N}(\{T\}), i \neq j}\left\|S^{j}-S^{i}\right\|_{M(i, j)}^{2}\right)}$, with $S^{i}$ (resp. $\left.S^{j}\right)$ being the point corresponding to node $i$ (resp. $j$ ),

- $c_{1}$ is the number of edges in a simplex, $c_{1}=\frac{d(d+1)}{2}$.

In this definition $M$ is a simplex-wise linear metric field which drives anisotropic mesh adaptation, $M(T)$ is the interpolation of $M$ at the center of $T$, and $M(i, j)$ is the interpolation of $M$ at the center of edge $(i, j)$. To prevent from confusion, it is important to mention that opposed to the term face that was used so far to refer to an edge in $2 \mathrm{D}$ and a face in $3 \mathrm{D}$, the word edge refers here to an edge in both dimensions. In the general anisotropic case, $M$ takes the form:

$$
M=R\left(\begin{array}{ccc}
\frac{1}{h_{1}^{2}} & & \\
& \ddots & \\
& & \frac{1}{h_{d}^{2}}
\end{array}\right) R^{T}
$$

The matrix $R$ is a rotation matrix, and the values $h_{i}$ are the mesh sizes prescribed in the directions defined by the column vectors of $R$. The norm of a given vector $v$ can be obtained through the following formula: $\|v\|_{M}=\sqrt{v^{T} M v}$. Therefore, in the term $c_{0} \frac{|T|_{M}}{h_{M}^{d}}$ in Eq. (1), the Euclidean space is distorted so that the mesh adaptation tool sees an isotropic mesh with all edges of length 1 , while in the real space the mesh is anisotropic with various edge lengths as defined by the metric $M$. The second term $h_{M}^{d}$, resp. the third one $\frac{1}{h_{M}^{d}}$, in Eq. (1) penalizes the quality for too small, resp. too large, elements and enables mesh coarsening, resp. mesh refinement. All these definitions and the reasoning behind their choice can be found in [17]. Finally, the anisotropic mesh adaptation algorithm implemented in Fitz consists in browsing all nodes and 
faces of the mesh, collecting their neighborhood, and trying to replace it by a "better" set of simplexes. More particularly, starting from a given set of elements $\mathcal{T}$ constituting the neighborhood of a node or face of the mesh, it is tested whether connecting any node $n^{\prime}$ of the given set to all the nodes of the faces of this set that do not contain $n^{\prime}$ would lead to a "better" set of simplexes. When such a set is found, it replaces the original set if and only if the two sets have the same volume. This last condition ensures mesh conformity (in a FE sense). The operation which consists in connecting a node to all possible faces is usually called star-connecting in the literature. The whole algorithm can hence be seen as a star-connecting optimization process. Node creation can be inserted in this algorithm by also considering the barycenter of $\mathcal{N}(\mathcal{T})$ as a candidate in the process.
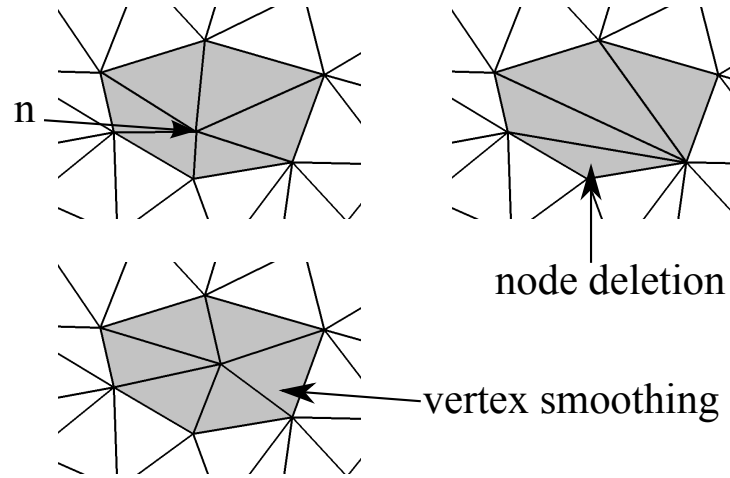

(a)
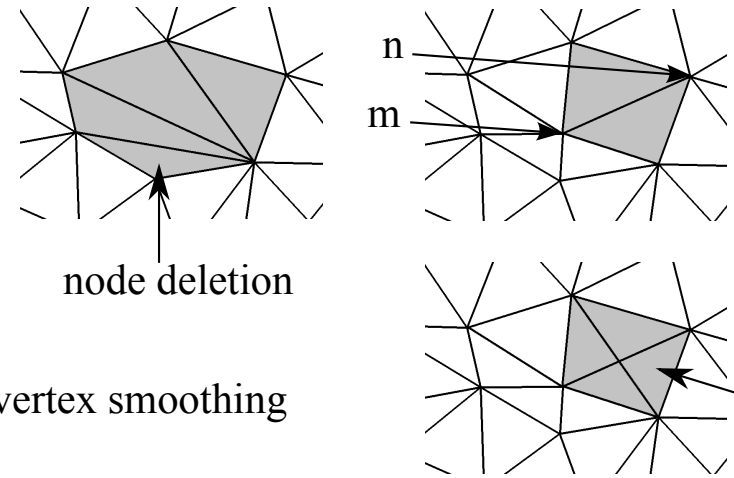

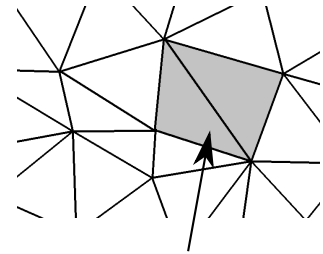

edge swapping

\section{node creation}

(b)

Figure 2: Examples of local mesh adaptation operations performed by the star-connecting optimization process.

In other mesh adaptation techniques, several adaptation operations are defined, such as edge splitting, edge collapse, vertex smoothing, edge swapping, etc., priorities have to be set between them and some particular cases such as infinite loops have to be handled [23, 24]. It can be easily seen that all these operations are included in the star-connecting optimization algorithm, which basically considers all possibilities. Some of these equivalencies are illustrated in Fig. 2, where the neighborhood is colored in light gray. In (a), two possible modifications are shown for a node: it can be deleted by star-connecting the neighborhood to a different node, or it can be smoothed by star-connecting the neighborhood to its barycenter. In (b), two possible modifications are shown for a face: it can be swapped by star-connecting the neighborhood to a different node, or a new node can be created by star-connecting the neighborhood to its barycenter. Illustrating all possibilities would be irrelevant and too long here, as they increase with new geometries and of course in 3D. Nevertheless, these simple examples show the large variety of topologies visited by the optimization algorithm. In the following, a focus is made on the new operations that were introduced in Fitz to address the (re)meshing and preservation of internal surfaces.

\subsubsection{Interface fitting}

The first step is to "bring" the mesh on the interface. Such operation is already possible in some commercial codes. Having an initial volume mesh of an object, one can introduce another object, and the software will:

- localize in the initial mesh the simplexes which are crossed by the surface of the new object;

- split these simplexes in order to follow the surface mesh of the new object;

- adapt the mesh inside the two objects.

The last point is optional, however when splitting simplexes in the second step, the resulting mesh may be of poor quality in some areas. Since in the present application, objects will be introduced several times during the simulation to model void coalescence, the localization step may be too expensive. Moreover, it is important here to remind that the framework is based on a LS representation of interfaces. That is the 
reason why a methodology which is based on the cut-cell method is preferred [28]. In this methodology, the implicit representation of interfaces is used to cut the cells crossed by the interface. The difference with a classical cut-cell method is the presence of anisotropic mesh adaptation before and after cell-cutting.

Let $\psi$ be the LS function representing the void/matrix interface. Before considering any fitting of the mesh on the interface, it is important to have a mesh fine enough close to the zero level of $\psi$. To obtain this result, mesh is adapted using the anisotropic mesh adaptation algorithm presented earlier together with the metric field detailed in [29]. This metric field is based on an a priori error estimator linking the interpolation error on the LS function to its gradient vector and hessian matrix. These variables represent respectively the normal vector to the interface and its main curvatures. Using this data, a metric field can be built in order to have a very fine mesh in the normal direction to the interface, and to control the mesh size in the other directions depending on local curvatures. In practice, the signed distance function is first computed, and then the metric field and finally remeshing is operated. This whole LS/metric/remeshing process is iterated several times because depending on the initial mesh, the first computations of the metric field may be inaccurate.

An example of mesh obtained using this methodology is illustrated in Fig. 3, where all local variations of the interface are progressively captured. Five LS/metric/remeshing iterations are performed from the initial state shown in Fig. 3(a), to the final state shown in Fig. 3(c), where the letters of Fitz can be totally and accurately recognized. In this final state, an average simplex quality close to 0.8 was obtained, which serves as an indicator of convergence. Regarding computation time, some measurements on real cases will be presented in section 4.2 .

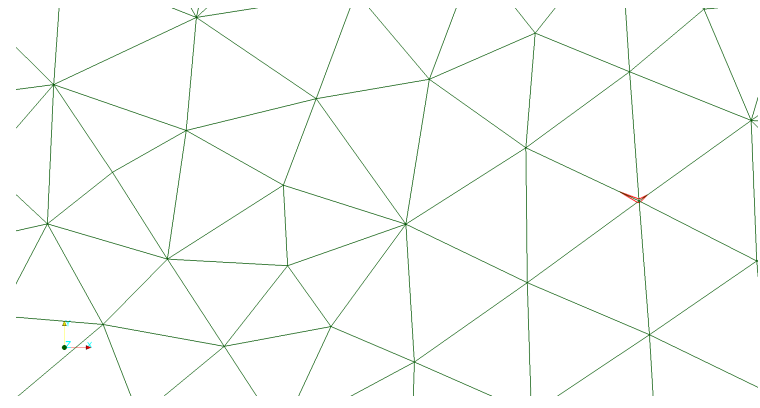

(a)

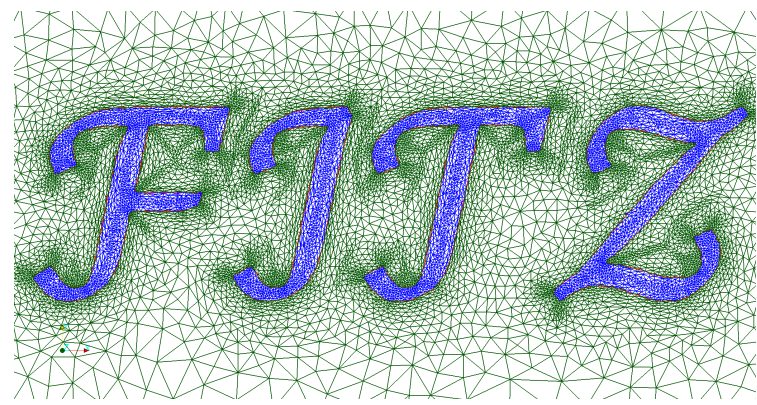

(c)

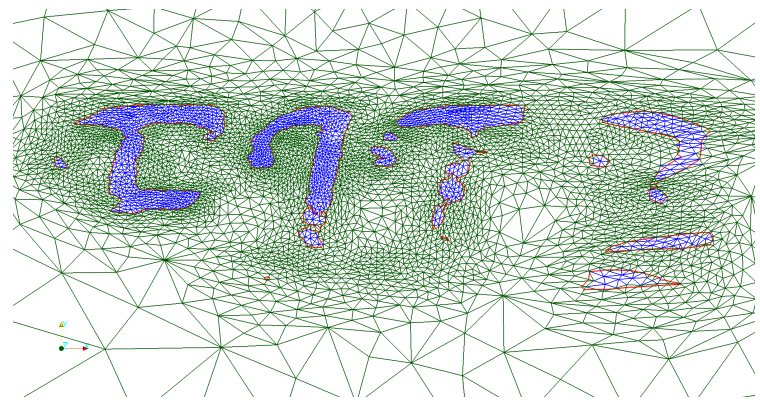

(b)

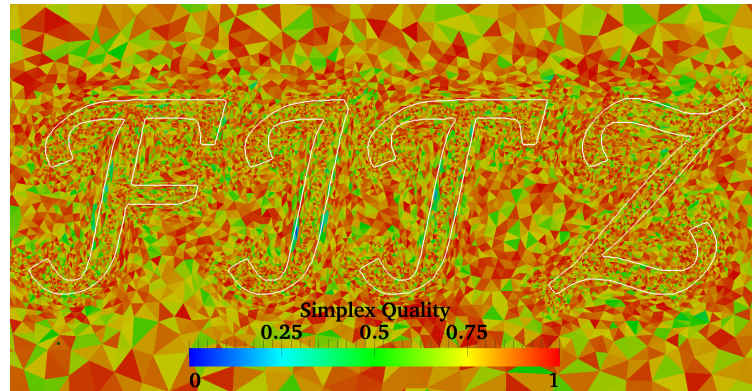

(d)

Figure 3: Mesh adaptation using Fitz from an isotropic mesh (a), to an anisotropic mesh adapted to the interface (b), (c). The interface is represented in red. In (d), the quality of the resulting mesh is shown, with the interface colored in white.

Once a satisfying mesh is obtained close to the interface, fitting can be operated. As illustrated in Fig. 5(b), this operation consists in browsing all edges of the mesh and splitting into two the edges where $\psi$ takes two different signs. At such edges, the intersection $S^{i j}$ between the interface and the edge $(i, j)$ is obtained by means of linear interpolation:

$$
S^{i j}=S^{i}-\left(S^{i}-S^{j}\right) \frac{\psi\left(S^{i}\right)}{\psi\left(S^{i}\right)-\psi\left(S^{j}\right)} .
$$


Finally, the parallel implementation of CimLib implies that the mesh is distributed between the various processes, which then only have the knowledge of a partition of the mesh. Regarding remeshing, it is applied independently by each process, inter-partition boundaries being blocked. Then, repartitioning is performed so that poor quality elements land far away from these inter-partition boundaries, using the methodology introduced in [16], and remeshing is applied independently again. Regarding fitting, it is not performed on inter-partition boundaries, but mesh quality is penalized for unfitted elements so that they can be fitted after repartitioning.

\subsubsection{Interface locking, refining and coarsening}

Obviously, fitting the mesh on the interface would be worthless if the mesh adaptation process was allowed to remove nodes from the interface without any regulation. Thus, locking the interface is necessary. Nevertheless, it was mentioned earlier that it is also important to enable interface remeshing to prevent poor quality elements from appearing close to the interface.

To enable both interface locking and remeshing, faces that carry the interface are collected in the starconnecting optimization process. If such faces are found inside a set of elements, then node creation is constrained by replacing the neighborhood barycenter by the barycenter of each face. Node deletion and star-reconnection are also constrained by only enabling reconnection to a node where the LS function is null.

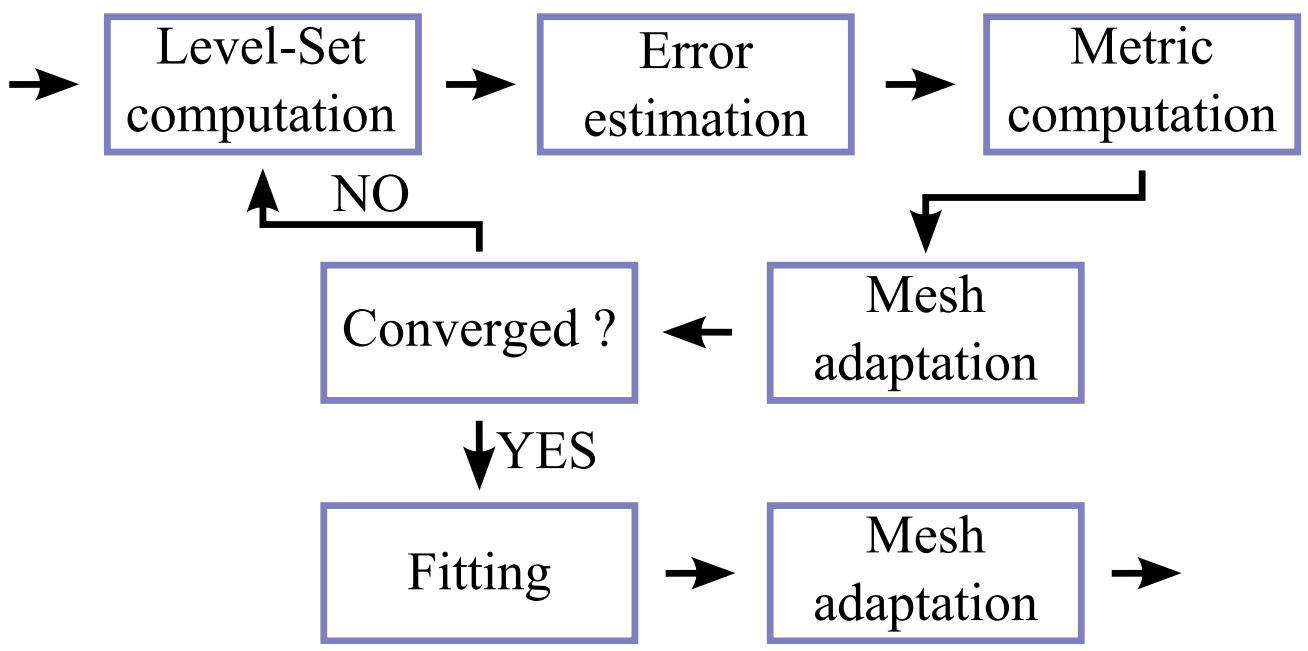

Figure 4: Flow chart of the remeshing methodology.

The flow chart in Fig. 4 summarizes this new body-fitted approach. Any new object to be inserted in the immersed volume simulation is first represented by its LS function $\psi$, which is a signed distance function to this object's boundary. Then a metric field $M$ is built to refine the mesh close to this object's boundary. The global star-connecting optimization process is then executed and results in Fig. 5(a). Distance computation, metric construction and mesh adaptation may be performed several times to converge to an accurate mesh close to the interface as in Fig. 3. The interface can be fitted on this mesh by splitting elements, as illustrated in Fig. 5(b). Finally, remeshing can be operated in the volume as on the surface, with the constraint that it has to preserve the surface, as seen in Fig. 5(c). It can be observed in this last figure that interface remeshing may change locally the shape of the interface. A particular attention is given to this effect in section 4.1.3. 


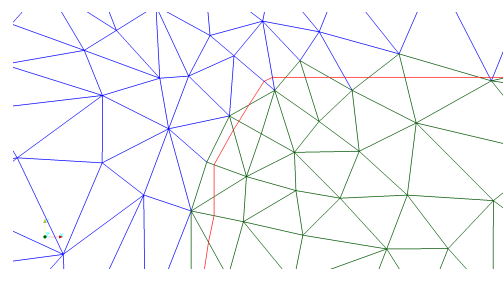

(a)

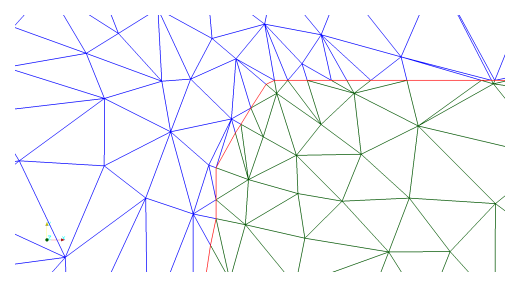

(b)

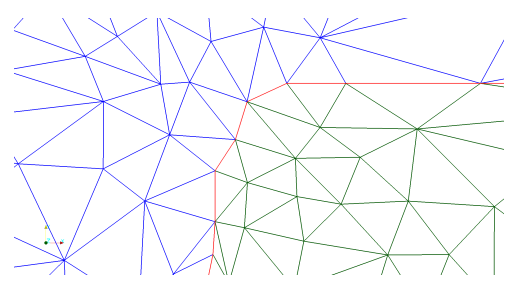

(c)

Figure 5: Different steps of the new body-fitted approach, the matrix phase is colored in green, the void in blue, and the red curve is the interface.

\subsection{Mesh adaptation to the mechanical fields}

So far, the P1 metric field $M$ was defined to have an accurate description of interfaces. In practice, an accurate description of mechanical fields is also required, as they drive void growth and coalescence. Similarly to the approach used to refine the mesh close to the interfaces, an error estimator is first needed. Here, an a posteriori error estimator based on the interpolation error on the plastic strain rate $\dot{\bar{\varepsilon}}$ is used. Since this variable is constant per element, its interpolation error is given by its gradient, which can be computed directly only for P1 variables. To recover an element-wise gradient, the methodology used here consists in first recovering a higher order approximation of the plastic strain rate on the nodes, and then computing the element-wise gradient of this P1 field.

The higher order approximation is obtained using the modified Superconvergent Patch Recovery (SPR) technique presented in [30]. On each node, this technique consists in collecting the values of the given variable on every neighboring element. Then, a second order Hermite interpolation of the variable is fitted on these values in a least square sense. A common issue with a high order SPR technique is the important number of unknowns: the value of the field, the $d$ values for its gradient, and the $d(d+1) / 2$ values for its hessian matrix. In order to well-define the least square problem, the patch must then contain enough data $(1+d+d(d+1) / 2)$. In the frame of the present work, a parallel algorithm was developed in the CimLib library to reach this purpose. As pictured in Fig. 6(a), this algorithm consists in first collecting two sets at every node of the mesh: the data, which contains the values of the field on the neighboring elements, and the ghost, which contains the nodes connected to the considered node. Until data does not contain $1+d+d(d+1) / 2$ elements, the two sets are fed by collecting the data and the ghost of the nodes already located in ghost, as shown in Fig. 6(b). In this implementation, the heterogeneities are not taken into account. In particular, data may contain elements of the void phase for matrix nodes close to the interface. This is not an issue here since close to the interface, there is another dominant error estimator, which is the one built thanks to the LS function. 


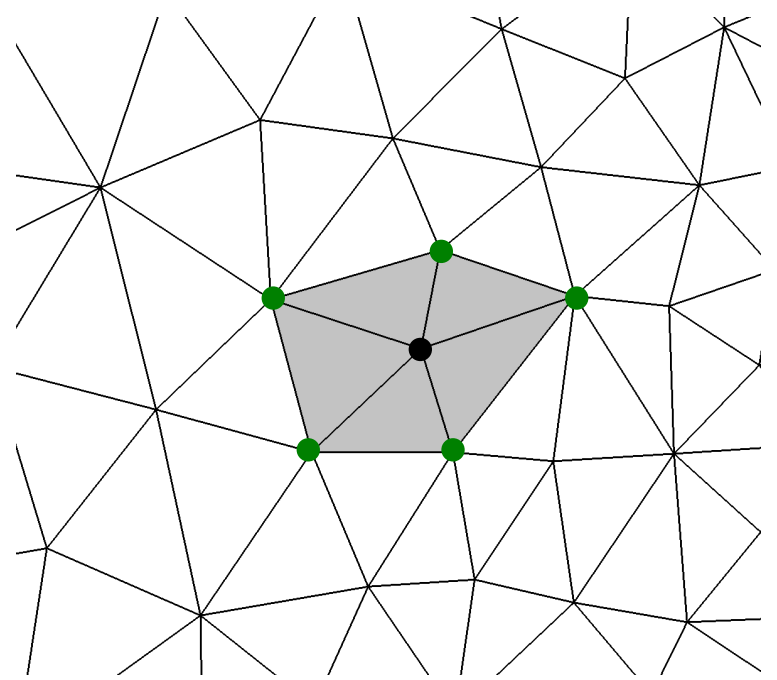

(a)

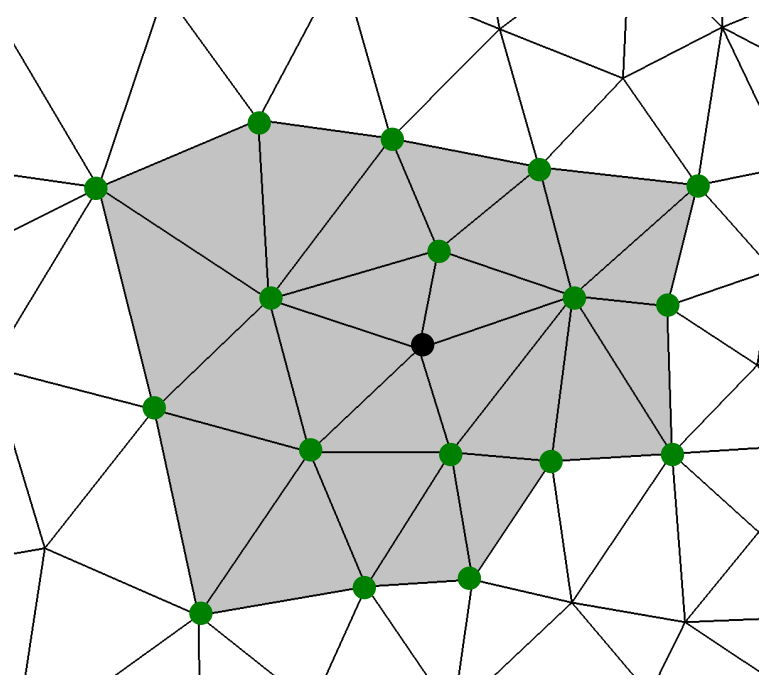

(b)

Figure 6: Illustration of the patch, or data (light gray) and the ghost (green dots) for a considered node (black dot) at the first (a) and second (b) iterations of the feeding algorithm.

Once the higher order approximation is recovered, its gradient can be computed element-wise and an isotropic mesh size is obtained:

$$
\forall T \in \mathcal{T}_{h}, h_{\nabla}=\max \left(\min \left(h \frac{\epsilon}{\|\nabla \dot{\bar{\varepsilon}}\|}, h_{\max }\right), h_{\text {min }}\right)
$$

where $\epsilon$ is a prescribed interpolation error and $h=\frac{1}{c_{1}} \sum_{(i, j) \in \mathcal{N}(\{T\}), i \neq j}\left\|S^{j}-S^{i}\right\|$ is the Euclidean average edge length, using the Euclidean norm $\|$.$\| . The maximal and minimal mesh sizes h_{\max }$ and $h_{\min }$ are used to prevent from inconsistent results when the gradient is equal to zero or ill-defined (e.g. near discontinuities). After coalescence, plasticity may localize in totally different regions than before. To prevent huge mesh changes at such moments, the ratio $h_{\nabla} / h$ is also controlled with an upper and a lower bound. The resulting mesh size $h_{\nabla}^{\prime}$ gives an element-wise metric field $M_{\nabla}=h_{\nabla}^{\prime}{ }^{-2} I$. To be used as an entry to Fitz, this metric field is brought at the nodes using once again the SPR technique.

To combine the mechanical metric field $M_{\nabla}$ with the one used to adapt the mesh to the interfaces, metric intersection is used. Briefly, this technique builds a unique metric always producing the largest mesh size satisfying any set of metrics given as input [31].

An example of mesh obtained by combining all mesh adaptation strategies mentioned in this paper is presented in Fig. 7. In this illustration, different scales are defined depending on the distance to the void/matrix interface. Far from the interface, an isotropic coarse mesh size is imposed. In an intermediary zone, linear transition is used to smoothly change to the fine mesh size imposed close to the interface. In this last zone, mesh is adapted to the interfaces and to the plastic strain rate. Arrows point out some areas where mesh can be clearly observed as adapted to the plastic strain rate. 


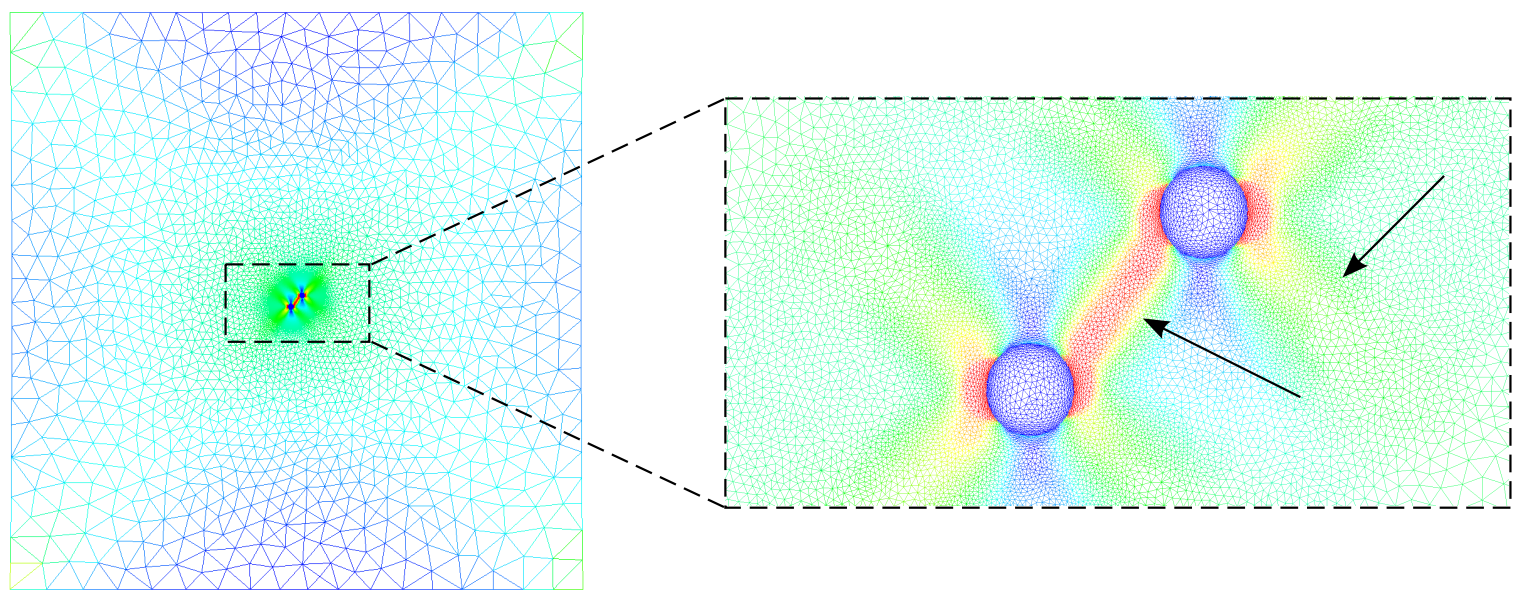

(a)

(b)

Figure 7: Example of mesh used for simulations. Here mesh size is adapted from the large scale of the domain (a) to the small scale of the two immersed voids (b). The color scale is based on $\dot{\bar{\varepsilon}}$.

\section{Mechanical modeling}

\subsection{Material behavior and mechanical formulation}

In the present body-fitted framework, each element of the mesh will either be a matrix element or a void element. Regarding the matrix, it will be considered as an elasto-plastic solid. Elasticity is ruled by the Hooke's law for isotropic linear elasticity, and plasticity by a von Mises yield criterion and an isotropic hardening law:

$$
\sigma_{0}(\bar{\varepsilon})=\sigma_{y}+K \bar{\varepsilon}^{n}
$$

Here $\bar{\varepsilon}$ is the accumulated plastic strain, $\sigma_{y}$ the yield stress, $K$ the plastic consistency and $n$ the hardening exponent. Numerical integration of this non-linear behavior is performed using a radial return mapping algorithm.

As in $[14,15]$, the void phase is considered as a compressible Newtonian fluid of dynamic viscosity $\eta_{\emptyset}$ and bulk viscosity $\chi \emptyset$ :

$$
\left\{\begin{array}{c}
\sigma=2 \eta_{\emptyset}\left(\dot{\varepsilon}-\frac{1}{3} \operatorname{tr}(\dot{\varepsilon})\right)-p I \\
p+\chi_{\emptyset} \operatorname{tr}(\dot{\varepsilon})=0
\end{array} .\right.
$$

This mixed velocity/pressure formulation is solved using a $\mathrm{P} 1+/ \mathrm{P} 1$ element on a single mesh, together with the formulation corresponding to the elasto-plastic phase (not recalled here). A Newton-Raphson algorithm is used to solve iteratively this formulation until convergence. More details on the $\mathrm{P} 1+/ \mathrm{P} 1$ element can be found in [32], and the formulation corresponding to the elasto-plastic phase and its resolution are presented for example in [33]. As already stated, an updated Lagrangian formulation is used, which avoids the resolution of any convection equation for the LS function. Nevertheless, it is important to adapt the mesh regularly to prevent from element flipping.

Finally, deforming the mesh is likely to distort the LS function, and since remeshing relies heavily on its gradient and hessian matrix, this may cause inconsistent remeshing. To prevent from such issues, the LS function is reinitialized, which means it is regularly brought back to a signed distance function to the interface during the simulation. The direct reinitialization technique proposed in [34] is chosen to perform this operation. 


\subsection{Modeling of void coalescence}

One the one hand, from an experimental point of view, the coalescence mechanism raises important issues. Any ductile material usually contains an initial volume of voids, which are parts of the defects of this material. Under loading, these voids may grow and other voids may appear due to void nucleation near intermetallic particles. However, both populations of voids are randomly distributed and hence difficult to observe $[35,36,37]$. Regarding the measurement of plastic strain and stress state at the microscale, recent developments in the field of Digital Volume Correlation (DVC) have led to promising results [38]. Nevertheless, the fact that void populations are random still remains an issue. In [39], Weck et al. have proposed to manually insert holes in test materials by means of laser drilling. Based on this technique, experiments were carried out with two or more voids, where void spacing and positioning could be controlled $[40,41]$.

One the other hand, from a modeling point of view, most works are based on FE studies [10, 11, 12]. A spherical or elliptic void is placed in a small Representative Volume Element (RVE), called unit cell. Periodic boundary conditions are used to simulate an infinite void array, and apply periodic homogenization theory. Opposed to experimental studies, FE calculations make it easier to conduct tests under various void arrangements, materials and stress states. Moreover, not only their influence on void volume evolution can be quantified, but the precise evolution of void shape can also be measured. However, due to implications on computational costs, multi-void RVEs with complex void arrangements and clusters are generally not studied in the literature. An exception can be found in [36], where the RVE was not an ideal void arrangement but a $2 \mathrm{D}$ mesh of a real microstructure obtained from imaging. In the present work, an infinite matrix will be simulated by including the RVE in a large domain, as already presented in Fig. 7. This approach cannot be compared to periodic homogenization, and has some limitations that will be discussed in section 4.2 . It is chosen here to avoid the difficulty of implementing periodic boundary conditions in an adaptive parallel FE framework.

Finally, most FE studies limit their scope to the prediction of void coalescence. Thus, the simulation only takes void growth into account, and it is stopped at the onset of void coalescence. Thanks to the body-fitted remeshing methodology developed here, the simulation can be carried out after void coalescence.

Void coalescence is usually defined as a significant acceleration in the evolution of void volume, due to the linking of voids at the microscale. To model this phenomenon, a criterion is to be defined to predict when linking of any pair of voids in the simulation will occur. If predicted, void coalescence will be operated by inserting a new void, called nucleus, in the simulation. An analysis of common void coalescence models such as the Thomason or McClintock models can be found in [40], where they were compared to experimental results. These criteria are based on void geometry, and macroscopic strain and stress fields. Moreover, these criteria address only ideal void shapes. Therefore, they do not correspond to the applications targeted by the present study. For example, the Thomason model does not seem to be applicable to the shape formed by two coalesced voids.

For these reasons, a different approach is developed, that does not cover all modes of void coalescence, especially when plastic localization occurs at a scale larger than void size, with negligible void nucleation and growth, as observed in [37]. Such phenomena are out of the scope of the present study, which focuses on modeling void coalescence in 2D materials with high void volume fraction, as in [40]. Here, it is assumed that the coalescence of the major voids modeled and meshed explicitly in the initial RVE is due to the linking of minor voids of a second population. These minor voids are supposed to be small enough to be homogenized in the behavior of the matrix. This approach has been used for example in [8] and [11] where the minor population was represented by a Gurson-type model, which also accounts for softening by modifying the plastic flow rule of the matrix. This raises new issues such as abnormal localization and softening dependency on the mesh that are generally solved by introducing one or multiple length scales in the plasticity-damage model [42]. Though these length scales are commonly accepted to be characteristics of the material, their determination would be tricky at the micron scale. That is the reason why an uncoupled Lemaitre damage model is preferred here. When the damage variable $D$ has reached a certain threshold $D_{c}$ on a whole line between two voids of the major population, coalescence is modeled as an instantaneous failure of the matrix between these two voids. This failure is supposed to be caused by the linking of voids of the minor population, as suggested in [41] for a copper alloy. Regarding the hypothesis of instantaneous 
failure, it is justified by the fact that no real measure of the velocity of crack propagation at the microscale has been performed yet. It is hence supposed that the characteristic time length for the propagation of a crack from a void to another is inferior to the time step used for simulations.

More precisely, damage evolution is given by the following Lemaitre model [43]:

$$
\dot{D}=\left\{\begin{array}{ll}
\dot{\bar{\varepsilon}}\left(-\frac{Y}{S_{0}}\right)^{b}, & D<1 \\
0, & D=1
\end{array}, Y=\frac{-1}{2 E(1-D)^{2}}\left[(1+\nu) \sigma: \sigma-\nu(\operatorname{tr} \sigma)^{2}\right]\right.
$$

where $Y$ is the damage strain energy release rate, $E$ the Young modulus, $\nu$ the Poisson coefficient, and $\sigma$ the Cauchy stress tensor. The two remaining coefficients $S_{0}$ and $b$ are material parameters. In practice, the extensions of the Lemaitre model regarding void closure effects and damage evolution under low triaxiality detailed in [43] are also used. Damage evolution and then the damage variable are computed after each mechanical resolution.

As a conclusion, the proposed coalescence model consists in considering all pair of interface nodes corresponding to two different voids, and verifying that $D>D_{c}$ on the whole line $\mathcal{L}$ between them. Note that the cost of this operation is affordable in $2 \mathrm{D}$, but optimization of this process will certainly be necessary for $3 \mathrm{D}$ computations. When this criterion is verified for any pair of candidate nodes, a nucleus is inserted between them. This nucleus has the shape of a thick line linking both nodes. The signed distance function $\psi_{n}$ to the boundary of such object can easily be computed:

$$
\forall x \in \Omega, \psi_{n}(x)=\xi-d(x, \mathcal{L})
$$

where $\xi$ is the line's thickness. The first step towards its insertion in the simulation is to modify the LS function $\psi$ of the void/matrix interface [15]:

$$
\tilde{\psi}=\max \left(\psi, \psi_{n}\right)
$$

The new LS function $\tilde{\psi}$ replaces the previous one and the new interface is meshed using the methodology developed in this work. Remeshing is also applied to avoid poor quality elements. A strong coupling is then used: the mechanical problem is solved again at the same time step, but with the new void topology, and the criterion is checked again. Nuclei insertion, remeshing, mechanical resolution and criterion computation are performed again until an equilibrium state is found when no coalescence is predicted. Then the simulation can proceed to the next time step, as illustrated in Fig. 8.

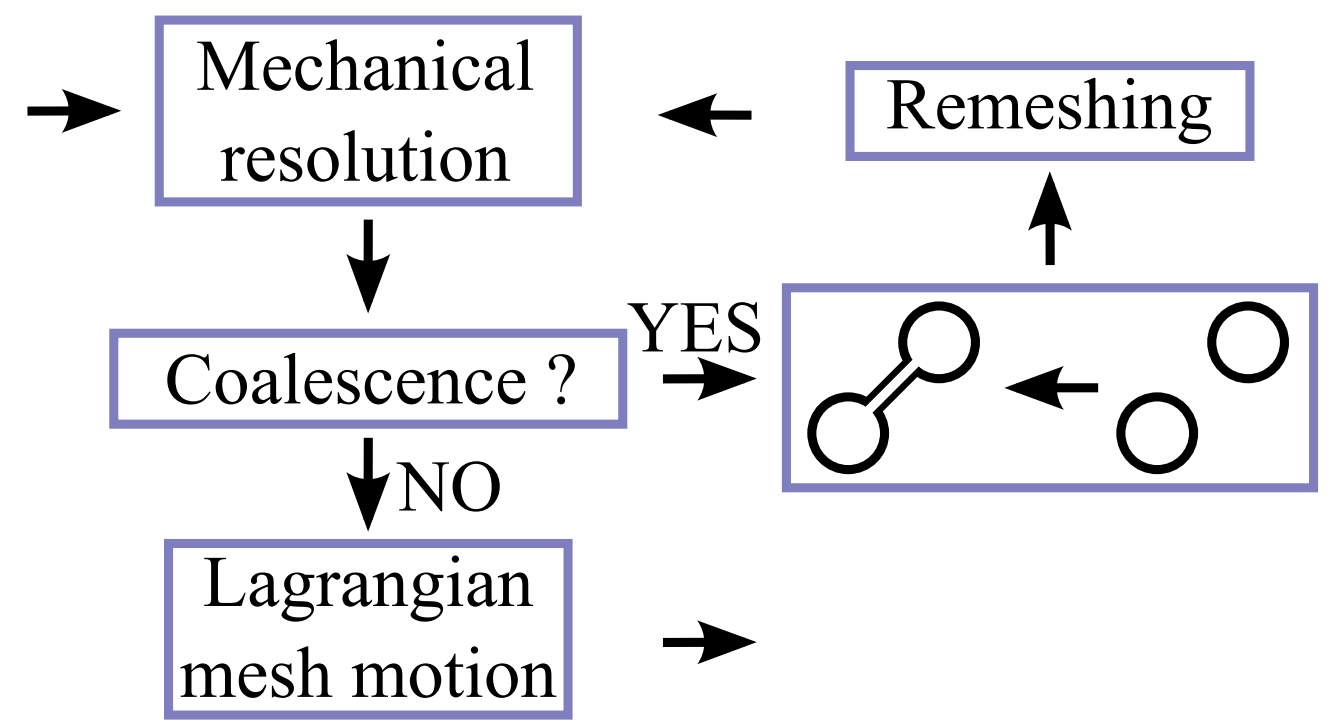

Figure 8: Strong coupling algorithm for void coalescence modeling. 


\section{Results}

The new body-fitted immersed volume method exhibits a certain number of differences with common approaches. More particularly regarding void coalescence prediction and modeling, the proposed model has to be confronted with experiments and observations found in the literature. This is the main purpose of the first part of this section. In a second part, the model is used to study the influence of relative void positioning and arrangement on ductility. The effect of stress state is also investigated. All these simulations are carried out in 2D under plane strain assumption. The extension to 3D would be possible with the same approach, but this would be more expensive for a large number of voids.

\subsection{Numerical validation}

Numerical validation is important to check that the proposed method gives consistent results when varying the numerical parameters. Here, four numerical parameters are to be investigated:

- domain size plays a major role in the modeling of an infinite matrix;

- mesh dependency is a common issue in all FE methods, so it is important that varying the mesh size does not alter the results;

- time step dependency is another problem that is theoretically avoided due to the implicit and strongly coupled solvers used in the present framework;

- rheology of the void phase has to be investigated to verify that it does not influence void growth or plasticity in the matrix.

The considered material is the aluminum alloy 5052 used in [40]. As stated earlier, this work is an experimental study of the loading of specimens containing laser drilled holes. The geometry of the test case chosen for validation is also issued from these experiments: two voids of identical radius $5 \mu \mathrm{m}$ and respective center positions $(-9.9 \mu \mathrm{m} ;-9.9 \mu \mathrm{m})$ and $(9.9 \mu \mathrm{m} ; 9.9 \mu \mathrm{m})$. It is illustrated in Fig. 9(a,b). Mesh size is quite an ambiguous notion in the present work due to the anisotropic mesh adaptation methodology being employed. In the large $10 \times 10 \mathrm{~mm}^{2}$ domain presently used, a first isotropic mesh size is defined. Then, in a closer region at a distance of $1 \mathrm{~mm}$ around the void/matrix interface, a transition to an intermediary mesh size is operated. Closer than $30 \mu \mathrm{m}$ to the interface, mesh is adapted to the plastic strain rate. Finally, closer than $4 \mu \mathrm{m}$, anisotropic mesh adaptation is used to accurately represent the interface. In Fig. 9(c), the lower and upper bounds for the mesh size are plotted in these different zones of the domain. Regarding the time step $\Delta t$, it corresponds to $0.01 \%$ of domain elongation per time increment. Material parameters defined in [44] are used and completed by standard values for an aluminum alloy concerning the Lemaitre model [43]. These values are summarized in Tab. 1. Moreover, the rheology of the void phase is described by $\eta_{\emptyset}=1 \mathrm{MPas}$ and $\chi_{\emptyset}=0.1 \mathrm{MPas}$. The loading is always tension in the vertical direction, with sticking upper and lower boundaries to favor necking. 


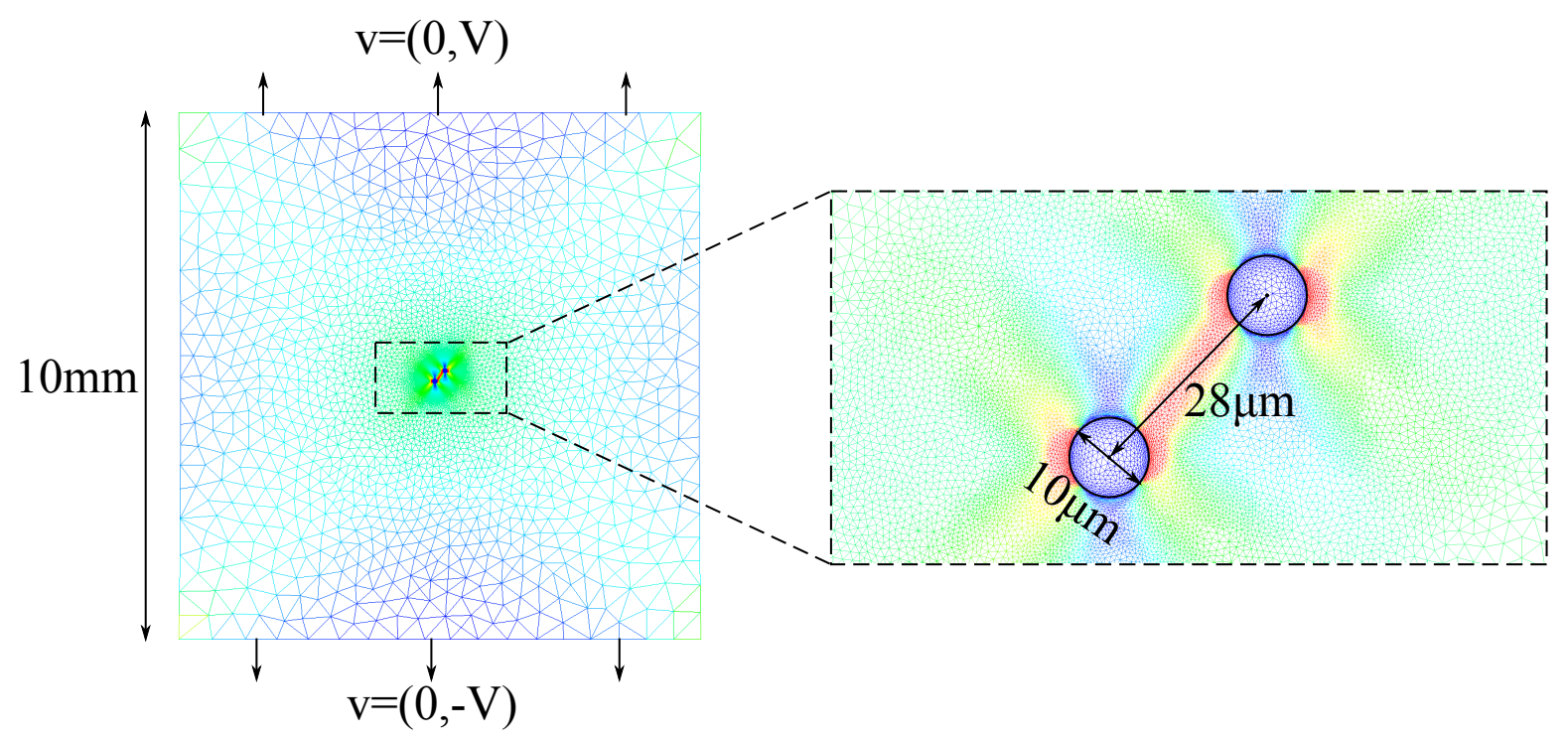

(a)

(b)

Figure 9: Reference computational set-up: (a) domain and loading configuration, (b) microstructure geometrical properties, (c) mesh sizes.

\begin{tabular}{ccccccc}
$E(\mathrm{MPa})$ & $\nu$ & $\sigma_{y}(\mathrm{MPa})$ & $K(\mathrm{MPa})$ & $n$ & $S_{0}(\mathrm{MPa})$ & $b$ \\
\hline 70000 & 0.33 & 0 & 604 & 0.3235 & 2.25 & 1
\end{tabular}

Table 1: Material parameters for AA5052 [43, 44].

\subsubsection{Influence of domain size}

In the present framework, a large domain is used to simulate an infinite matrix. To verify that this size is big enough, three simulations are run with square domains of sizes $1 \times 1 \mathrm{~mm}^{2}, 5 \times 5 \mathrm{~mm}^{2}$ and $10 \times 10$ $\mathrm{mm}^{2}$. At $20 \%$ of elongation of the domain, the total volume of the void phase $V_{\emptyset}$ is computed to quantify the influence of domain size on void growth. Regarding its influence on void coalescence prediction, the accumulated plastic strain $\bar{\varepsilon}$ is measured at the center of the RVE, which is located exactly between the two voids. As observed in [40], the coalescence crack is to go through this point. Void phase viscosities are fixed at $\eta_{\emptyset}=1 \mathrm{MPas}$ and $\chi_{\emptyset}=10^{-1} \mathrm{MPas}$ (see following section). The results are presented in Tab. 2, where the notation $\mathbb{E}(X)$ is used to represent the relative error in variable $X$ with respect to the value found for the same variable using the larger domain.

\begin{tabular}{ccccc} 
RVE size $\left(\mathrm{mm}^{2}\right)$ & $V_{\emptyset}\left(\mu^{2}\right)$ & $\mathbb{E}\left(V_{\emptyset}\right)$ & $\bar{\varepsilon}$ & $\mathbb{E}(\bar{\varepsilon})$ \\
\hline $1 \times 1$ & 492 & $3.93 \%$ & 0.710964 & $1.26 \%$ \\
$5 \times 5$ & 478 & $0.99 \%$ & 0.703354 & $0.18 \%$ \\
$10 \times 10$ & 473 & & 0.702125 &
\end{tabular}

Table 2: Numerical validation results regarding the influence of domain size.

The results illustrate that convergence is obtained both in terms of total void volume evolution and local strains. Though the errors obtained for a domain of intermediary size seem acceptable, they may increase for simulations with more complex void configurations. Hence, the larger domain is chosen for the all the following simulations. 


\subsubsection{Influence of void rheology}

Two material parameters were presented for the modeling of the compressible Newtonian behavior of the void phase: the dynamic viscosity $\eta_{\emptyset}$ and the bulk viscosity $\chi_{\emptyset}$. A sensibility analysis was already conducted in [14] regarding only the dynamic viscosity. In this section, this analysis is completed by including the bulk viscosity, and also by the measurement of the local strains. The results are presented in Tab. 3, using the same notations as in 4.1.1.

\begin{tabular}{cccccc}
$\eta_{\emptyset}(\mathrm{MPas})$ & $\chi_{\emptyset}(\mathrm{MPas})$ & $V_{\emptyset}\left(\mathrm{mm}^{2}\right)$ & $\mathbb{E}\left(V_{\emptyset}\right)$ & $\bar{\varepsilon}$ & $\mathbb{E}(\bar{\varepsilon})$ \\
\hline 1 & $10^{-1}$ & 477.64 & $0.06 \%$ & 0.702689 & $0.34 \%$ \\
$10^{-2}$ & $10^{-3}$ & 477.94 & & 0.705071 &
\end{tabular}

Table 3: Numerical validation results regarding the influence of void rheology.

Here only two sets of values are reported since no significant difference can be seen compared to the high ratio between viscosities. Due to the high discontinuities in stress/strain relations at the void/matrix interface, it is preferable to choose values as high as possible for the viscosities of the void phase to ease mechanical resolutions. Therefore, the first set of values is chosen for all following simulations.

\subsubsection{Influence of mesh size}

To study the influence of the meshing strategy on void growth and coalescence modeling, it is sufficient to only vary the characteristic mesh sizes in the regions where adaptive remeshing is used, which correspond to the first half of 9(c). Starting from the set of reference mesh sizes $h$, two new sets $h \times 2$ and $h / 2$ are built by respectively multiplying and dividing all the metric parameters by 2 in these regions (the metric itself is hence multiplied and divided by $2^{2}$ ). To also consider the influence of the volume of the nuclei inserted at the onset of void coalescence, the threshold $D_{c}$ is set at 0.5 and void coalescence is triggered when predicted. The thickness of the void coalescence crack $\xi$ is also multiplied and divided by 2 depending on the configuration. Here particular attention is given to the instant at which coalescence is predicted. Hence, the elongation at coalescence $l$ is reported in Tab. 4 together with the void volume at $30 \%$ of domain elongation for the three simulations.

\begin{tabular}{ccccc} 
Mesh size & $V_{\emptyset}\left(\mathrm{\mu m}^{2}\right)$ & $\mathbb{E}\left(V_{\emptyset}\right)$ & $l$ & $\mathbb{E}(l)$ \\
\hline$h \times 2$ & 2494 & $0.64 \%$ & $13.33 \%$ & $1.91 \%$ \\
$h$ & 2516 & $1.51 \%$ & $13.12 \%$ & $0.31 \%$ \\
$h / 2$ & 2478 & & $13.08 \%$ &
\end{tabular}

Table 4: Numerical validation results regarding the influence of mesh size. 


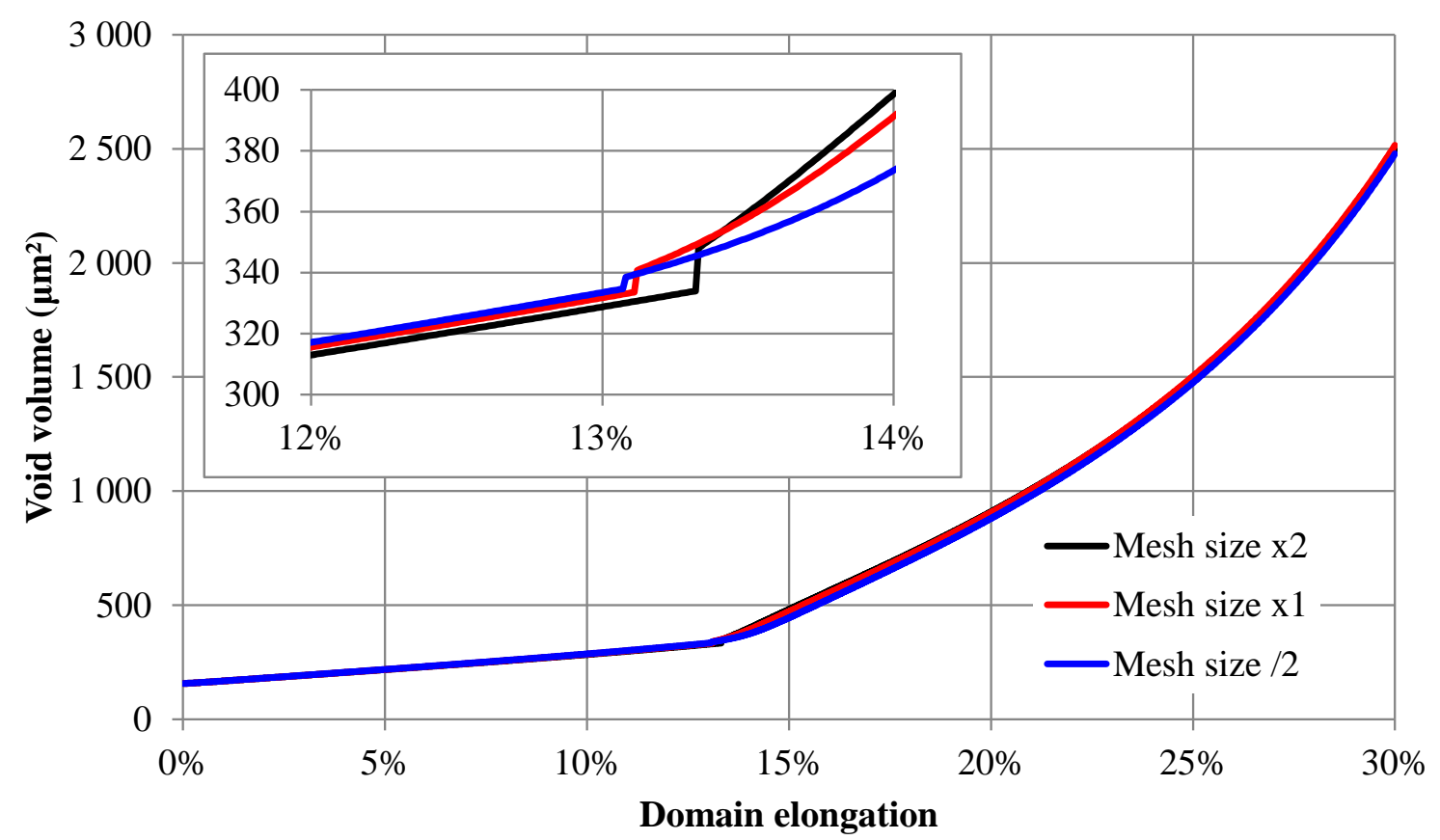

Figure 10: Void volume evolution for the three different sets of mesh sizes.

As shown in Tab. 4 and also in Fig. 10, the artificial volume of void inserted for the modeling of void coalescence is negligible compared to the volume gained by pure void growth. Regarding the void coalescence criteria, the results are consistent in terms of prediction of the onset and also in terms of prediction of the position and shape of the coalescence crack, as shown in Fig. 11(a). The important differences obtained in terms of void volume between the configurations are due to a better conservation of some sharp corners of the matrix depending on the used mesh, as presented in Fig. 11(b,d). Using the finest mesh, these portions of the matrix are accurately conserved through the simulation, while they are not captured with coarser meshes, thus the bigger void volume. For a better control of these anomalies, the variation of volume for each phase (void and matrix) should be quantified and reduced as much as possible during interface remeshing. Nevertheless, these simulations demonstrate the ability of the new body-fitted remesher to capture new interfaces on-the-fly during the simulation, with various remeshing configurations. 


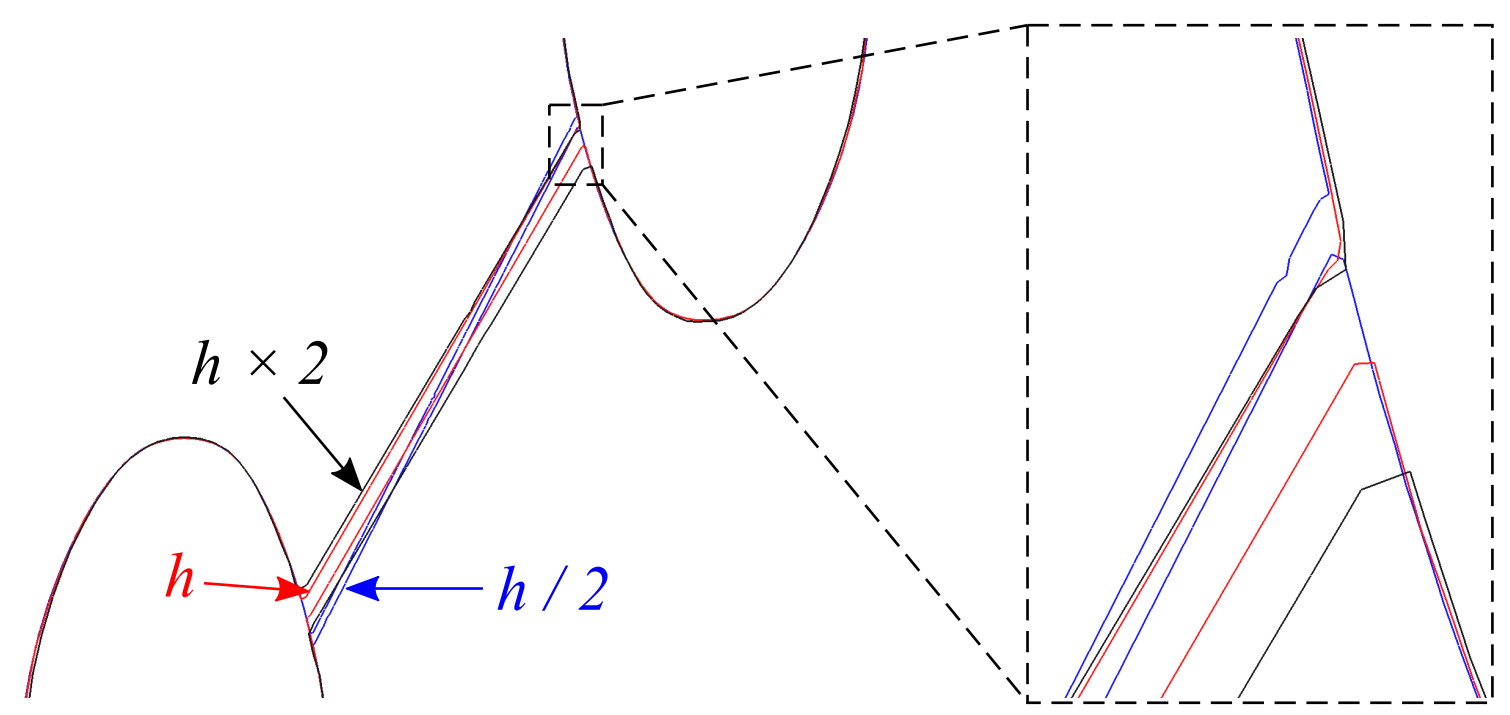

(a)

(b)

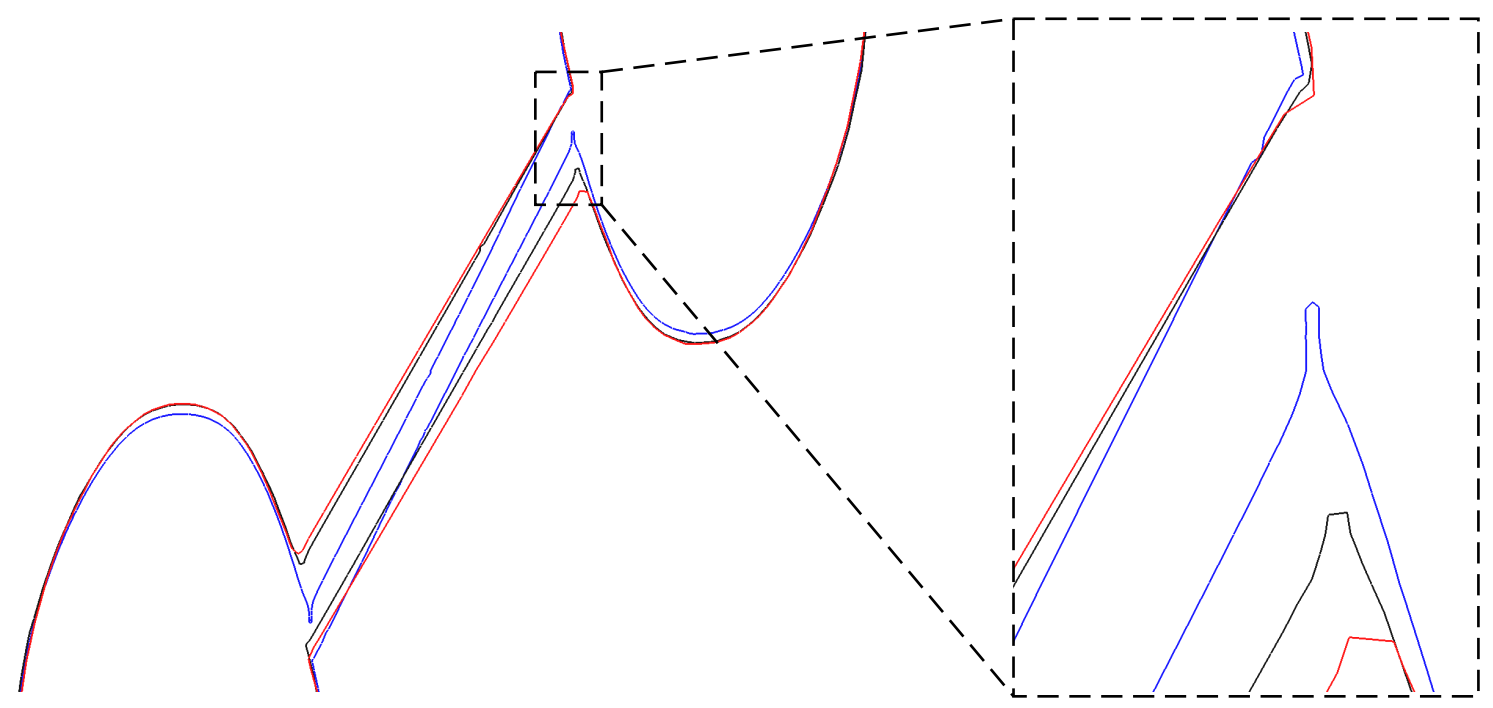

(c)

(d)

Figure 11: Shapes of the inserted nuclei at the respective onsets of void coalescence $(\mathrm{a}, \mathrm{b})$, and at $14 \%$ of domain elongation $(\mathrm{c}, \mathrm{d})$, for the three remeshing configurations.

\subsubsection{Influence of time step}

Based on the methodology used to solve the continuum mechanics equations and the nature of the void coalescence criteria, convergence is expected with a finer time step. For validation, three simulations are run with the time step $\Delta t$ used so far, a time step $\Delta t / 2$ two times smaller and a time step $\Delta t / 10$ ten times smaller. In order to also verify that repetitive remeshing does not produce excessive diffusion, remeshing is activated every ten iterations for the three simulations (hence there is more remeshing with a finer time step). As in 4.1.3, void coalescence is activated and the void volume at $20 \%$ of domain elongation is reported in Tab. 5 together with the elongation at the onset of coalescence. 


\begin{tabular}{ccccc} 
Time step & $V_{\emptyset}\left(\mathrm{\mu m}^{2}\right)$ & $\mathbb{E}\left(V_{\emptyset}\right)$ & $l$ & $\mathbb{E}(l)$ \\
\hline$\Delta t$ & 905.60 & $2.24 \%$ & $13.12 \%$ & $1.93 \%$ \\
$\Delta / 2$ & 903.22 & $1.97 \%$ & $13.20 \%$ & $1.33 \%$ \\
$\Delta t / 10$ & 885.78 & & $13.38 \%$ &
\end{tabular}

Table 5: Numerical validation results regarding the influence of time step.

No significant dependency on the time step can be observed in the results. On the one hand, the reference time step $\Delta t$ is already so small that reducing it does not produce significant differences in the results. On the other hand, increasing this time step leads to a divergence of the nonlinear solver because the plastic strain increment becomes too important at some points when coalescence occurs. Thus, this time step will be kept for all following simulations.

\subsection{Applications}

\subsubsection{Influence of relative void positioning}

In the following, the terms void orientation and relative positioning are indifferently used to describe the angle between the alignment direction of two circular voids and the loading direction. Depending on this angle, three modes of void coalescence are reported in the literature [13]: internal necking, shear coalescence and necklace coalescence. Shear coalescence is the phenomenon observed in the preceding simulations: plasticity localizes in a shear band between the two voids oriented at $45^{\circ}$ with respect to the loading direction, and coalescence occurs by failure of the matrix along this band. Internal necking is the most observed form of void coalescence: for two voids placed at $90^{\circ}$ with respect to loading direction, necking in the inter-void ligament leads also to plastic localization and to the apparition of a fracture plane perpendicular to the loading direction [40]. Necklace coalescence is a rare phenomenon that occurs when two voids are oriented along the loading direction. Until the onset of void coalescence the two voids grow towards each other, and a localization band appears between them. In this case, coalescence is often named void collapse, since at a critical instant, the two voids collapse into each other.

The following simulations test these three orientations using the present prediction criterion and modeling of void coalescence. In the three configurations, the voids have same radius and the distance between the centers is identical. The reference geometry is the one already used in 4.1, and the loading is also identical. The resulting void volume evolution curves are described in Fig. 12. The elongation at the onset of coalescence is of $10.74 \%$ for the $90^{\circ}$ configuration (against $13.12 \%$ for the $45^{\circ}$ configuration). These results are consistent with experimental observations made in [40]: "the sample with the holes oriented at $45^{\circ}$ is more ductile compared to those with the holes aligned at $90^{\circ}$ ". The shapes of the coalescence cracks are detailed on the same figure. They are consistent with the definitions given above regarding internal necking and shear coalescence. In the present modeling, the damage model is directly linked to plastic localization. Therefore, a correct prediction of the coalescence crack simply means that the plastic localization band was represented accurately enough. Such result is obtained thanks to the fact that the remeshing strategy takes the plastic strain rate into account. The rule that consists in checking that the coalescence criterion is verified on the whole localization band before triggering void coalescence is also crucial in order to have a coalescence crack included in this band. 


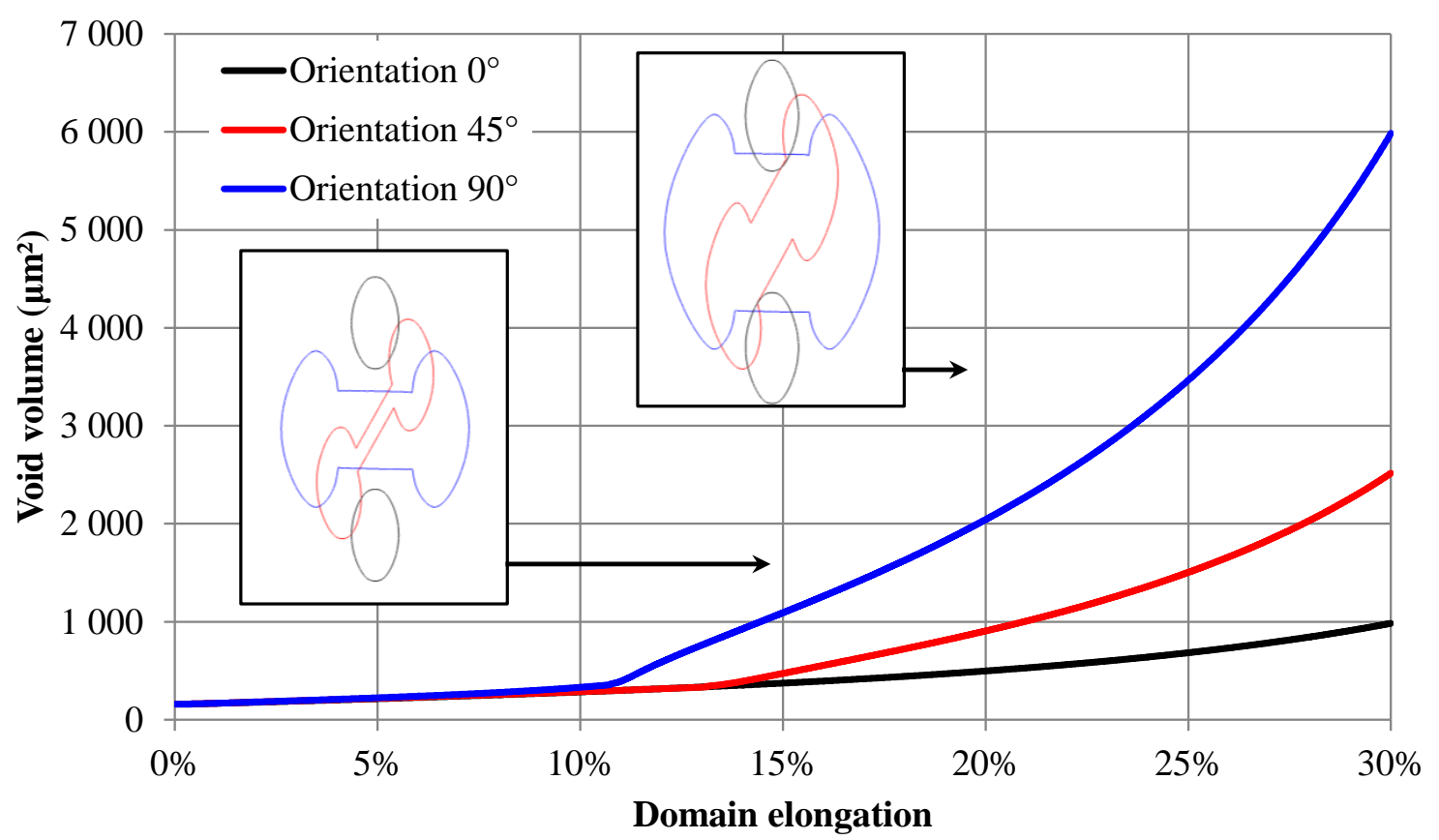

Figure 12: Void volume evolution for the three void orientations, and void shapes at $15 \%$ and $20 \%$ of domain elongation.

Regarding the configuration with voids aligned at $0^{\circ}$, no coalescence is predicted, which makes this configuration way more ductile than the two others. Pursuing the simulation to larger strain (45\%) reveals void growth in a diamond shape for both voids, still without any coalescence. This can be incriminated to the circular shape of the voids in the initial geometry. For necklace coalescence to occur, the voids need to be elongated in the loading direction. This will be investigated further in the following tests, which address multiple voids configurations.

\subsubsection{Influence of void arrangement}

The main advantage of the present framework is its applicability to random void clusters, and also to real void configurations. In the preceding section, void orientation was proved to have a major role on ductility. In this section, a larger number of voids are considered, and several periodic and random arrangements are tested to investigate the differences in ductility, and also on the propagation of local coalescence phenomena from one pair of voids to the others. Hence, the voids will always be circular with a unique radius of $5 \mu \mathrm{m}$.

Periodic arrangements. The two tested periodic arrangements contain respectively 36 and 38 voids oriented at $90^{\circ}$ and $45^{\circ}$. The spacing between void centers is constant and fixed at $37 \mu \mathrm{m}$. In Fig. 13, it can be observed that coalescence starts at the voids located at the top or the bottom of the microstructure and then propagates in a direction which is overall perpendicular to the loading direction. This propagation relaxes the stresses in the center of the RVE, where no coalescence is predicted. Domain elongation is of $11.48 \%$ at the onset of coalescence for the $90^{\circ}$ configuration and $13.50 \%$ for the $45^{\circ}$ configuration, as illustrated respectively in Fig. 13(b,c) and Fig. 13(f,g). The strong coupling algorithm presented in Fig. 8 plays a major role here since at some instants multiple coalescence cracks appear at the same time iteration. Additionally, these two simulations with arrays of holes give results which are consistent with the results obtained using only two voids, both in terms of ductility and mode of void coalescence.

However, they differ from results obtained using unit cell calculations in the literature and also from experimental observations. In a unit cell calculation, all voids would have coalesced identically, for example along an infinite repetition of straight horizontal lines for the $90^{\circ}$ configuration. Regarding the experiments 
performed in [40], a unique coalescence band was obtained (no periodicity), that crossed the RVE from left to right for the $90^{\circ}$ configuration, and from top left to bottom right for the $45^{\circ}$ configuration. Here, the RVE is included in an infinite non-softening matrix, and this approach matches neither unit cell calculations nor experiments. This is evidenced by the fact that for both configurations presented in Fig. 13, coalescence occurs only at the top and bottom voids of the arrangement. More particularly, the damage variable at the pre-coalescence instants pictured in Fig. 13(b) and Fig. 13(f) is already different for these "extreme" holes. Isolating these holes, it appears that opposed to the holes located in the center of the arrangement, they are trapped between a rigid matrix and a softening material composed of a matrix and holes. This heterogeneity locally increases plasticity and the stress state imposed to the RVE drifts from the periodic case, and is closer to the experimental one. It does not exactly match the experimental conditions because the full $3 \mathrm{D}$ specimen is not modeled. These limitations are to be investigated in the future. Regarding the influence of void clusters, it can still be studied, keeping in mind that void growth and coalescence are overestimated for voids located at RVE boundaries.

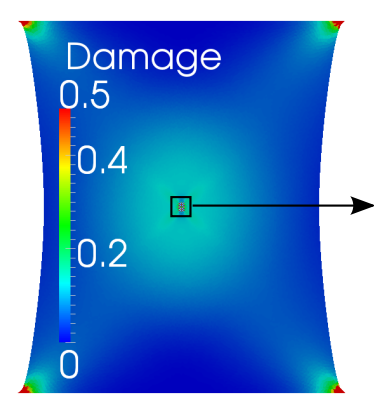

(a)

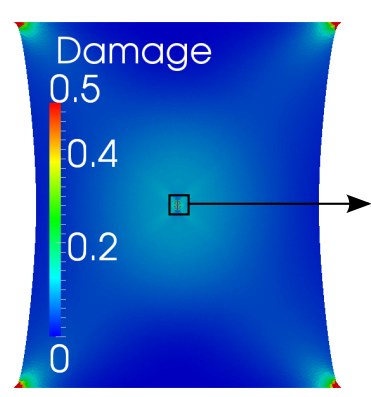

(e)

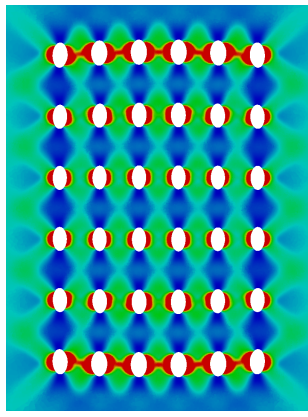

(b)

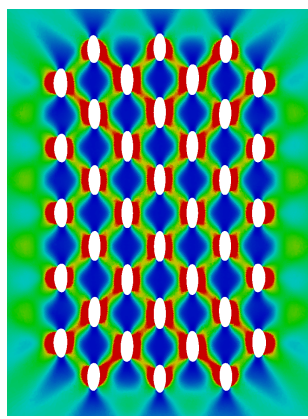

(f)

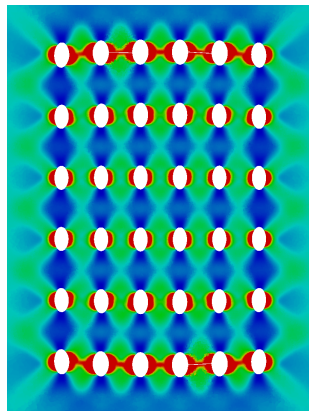

(c)

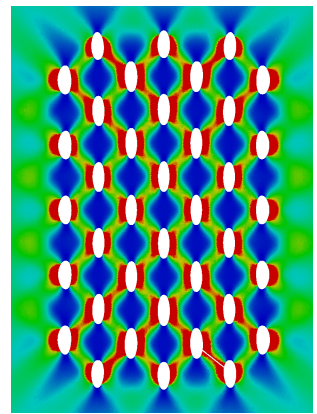

(g)

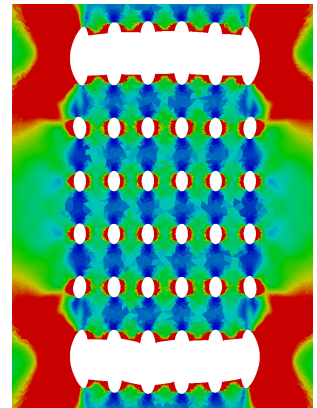

(d)

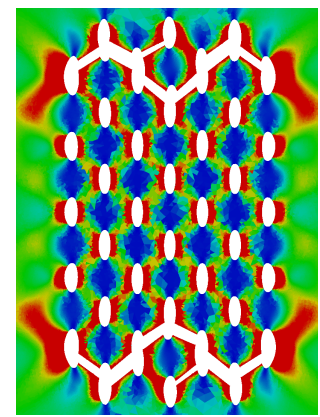

(h)

Figure 13: Ductile fracture prediction for the $90^{\circ}$ (top) and the $45^{\circ}$ (bottom) void arrays: (a,e) RVEs at the respective onsets of void coalescence, $(b, f)$ arrangements just before and, $(c, g)$, at the respective onsets of void coalescence, and $(\mathrm{d}, \mathrm{h})$ at $15 \%$ of domain elongation. Voids are not represented (white holes) and the color scale is based on the damage variable.

Random arrangements. Five random arrangements are generated using a Gaussian distribution of mean $20 \mu \mathrm{m}$ and standard deviation $10 \mu \mathrm{m}$. The number of voids, and hence the initial void volume in the RVE, is constant and fixed at 38 voids. The initial configurations and the obtained results during loading are presented in Fig. 14. Clearly, these microstructures are composed of void clusters of various sizes. Though coalescence is likely to occur where voids are close to each other and oriented perpendicular to the loading direction (as observed in the preceding section), it propagates in priority to voids of the same cluster. This can clearly be seen at the lower left corner of arrangement 0 at $7.5 \%$ of elongation. Then, coalescence can propagate to isolated voids or between clusters, as observed for the same arrangement at $12.5 \%$ and $17.5 \%$ of elongation.

Orientation still plays a major role here. In arrangement 1, a cluster of numerous voids located close to 
each other can be seen on the left of the microstructure, but no coalescence occurs in that cluster due to the vertical alignment. This is also observed in arrangement 3. On the contrary, arrangement 2 seems less ductile due to horizontal alignment. This can be already observed at $7.5 \%$ of elongation, where this arrangement has the larger void volume compared to the other configurations.

Ductility is further described in Fig. 15 which shows void volume evolution for the five arrangements. The curves confirm that arrangement 2 is less ductile and is subject to coalescence earlier than other arrangements. Regarding the most ductile arrangements 1 and 3, the effects of void clustering can be seen at large strain. While coalescence propagation is difficult in arrangement 1 for the reasons mentioned above, small clusters of voids located really close to each other maintain void interaction in arrangement 3 . That is the reason why void volume increase is more important for arrangement 3 in Fig. 15. Though the inter-void distance was higher for the two periodic configurations, their void volume evolution curves are reported on the same figure for comparison. Both periodic configurations are more ductile, which is due to the higher inter-void distance. Regarding void coalescence, the fact that it occurs earlier for random arrangements is mostly due to the presence of void clusters, where coalescence is premature because of lower inter-void distance. 

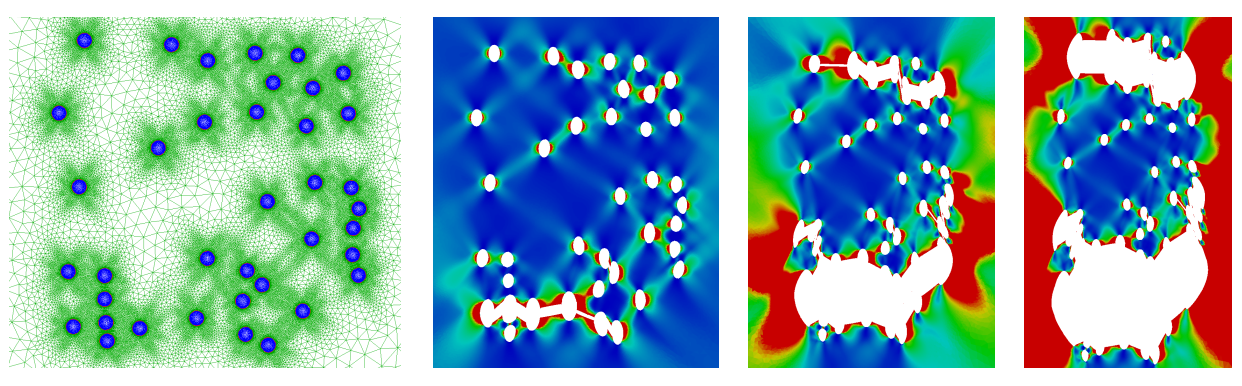

Ar. 0
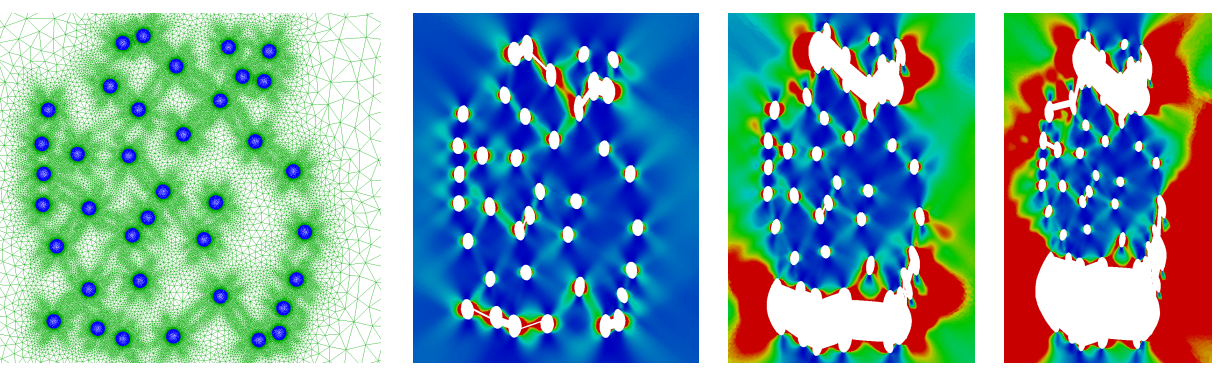

Ar. 1
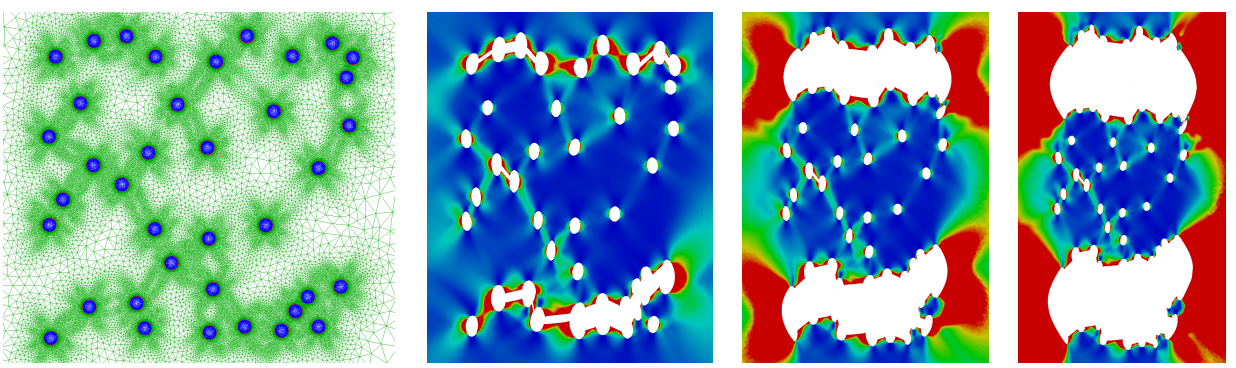

Ar. 2
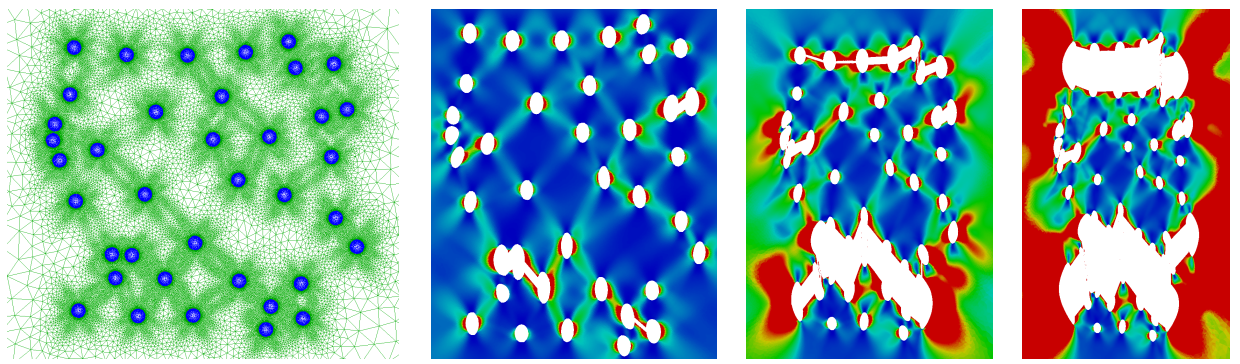

Ar. 3
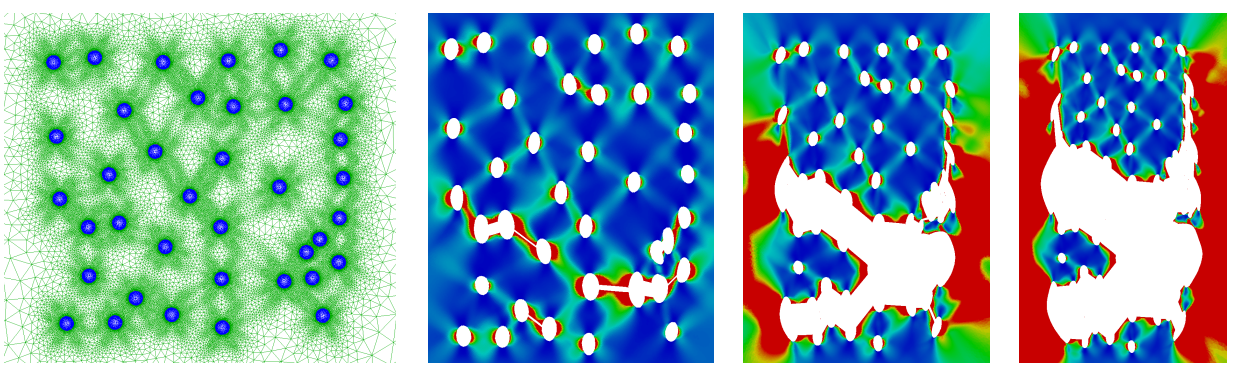

Ar. 4

$$
0.0 \%
$$

$7.5 \%$

$12.5 \%$

$17.5 \%$

Figure 14: Mechanical states of the five random void arrangements at various domain elongations. The mesh inside the voids is only represented in the first picture of each line. On the other pictures, the colors represent the Lemaitre damage field with the same scale as in Fig. 13. 


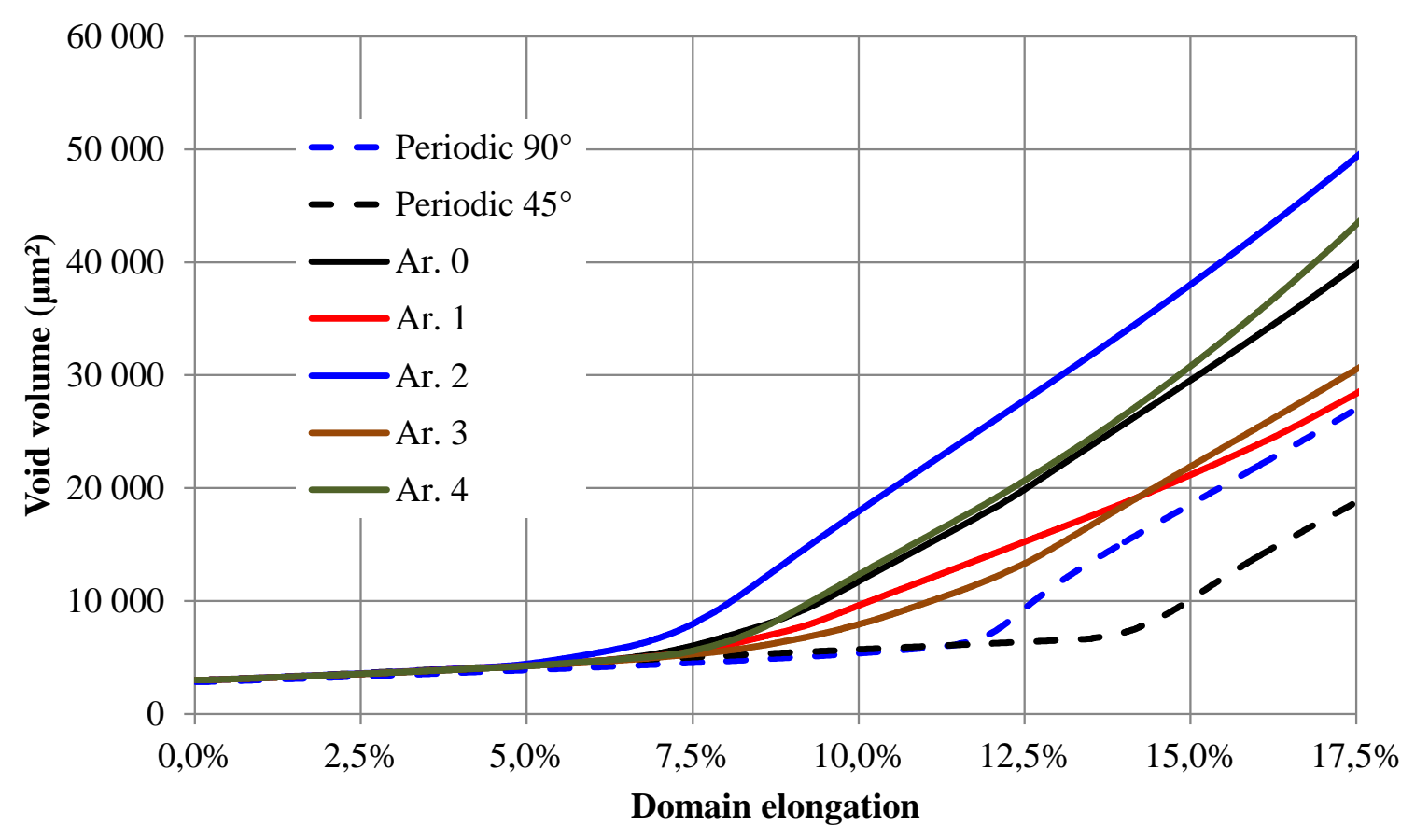

Figure 15: Void volume evolution for the two periodic void configurations and the five random void arrangements.

\subsubsection{Influence of stress state}

The influence of stress state on void growth has been repetitively studied in literature. Defining the triaxiality ratio as $T=\operatorname{tr}(\sigma) / \sigma_{0}$, it is well known that a higher triaxiality favors void growth and coalescence [45]. The aim of the present simulations is to observe this effect after the onset of void coalescence. At the upper and lower boundaries, velocity is imposed only in the vertical direction, the other velocity component being free. On lateral boundaries, a homogeneous normal stress is imposed and the triaxiality is measured. To perform this measure, simulations are carried out until $25 \%$ of domain elongation without any microstructure and the obtained stress state is hence homogeneous in the RVE, though it varies during loading. The configurations are: $T_{0}=0.57 \rightarrow 0.58$ (plane strain uniaxial tension), with a normal stress of $0 \mathrm{MPa}, T_{-}=-0.15 \rightarrow 0.32$, with a normal stress of $-100 \mathrm{MPa}$, and $T_{+}=1.32 \rightarrow 0.84$, with a normal stress of $100 \mathrm{MPa}$. The variations are due to the fact that the applied normal stresses remain constant during loading, while they should vary to keep the triaxiality constant. This is not an issue here as long as the obtained triaxiality ranges do not overlap. The microstructure used for these tests is the arrangement 4 of the preceding section. 


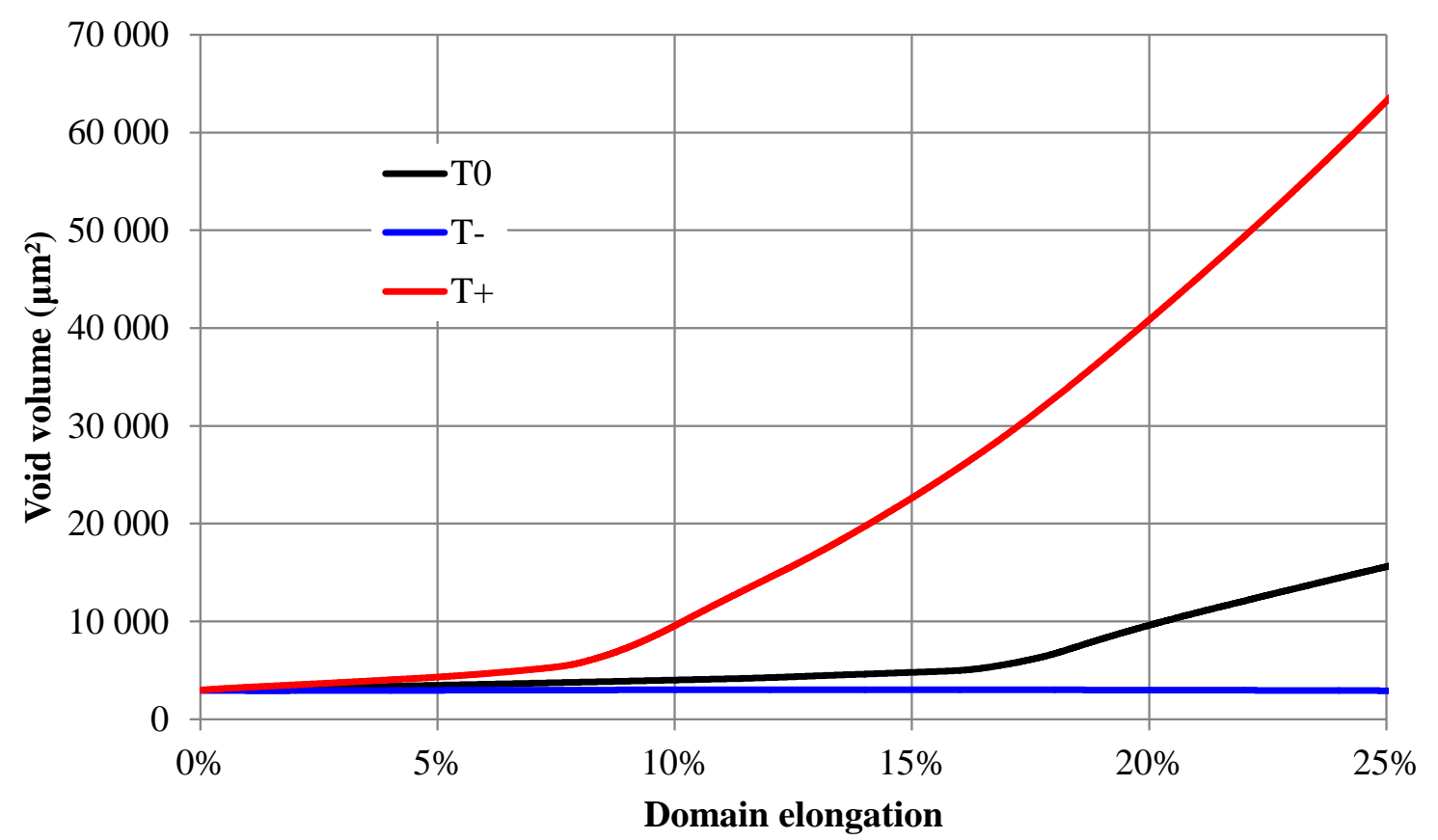

Figure 16: Void volume evolution for the three different stress states: uniaxial tension $T_{0}$, low triaxiality $T_{-}$, and high triaxiality $T_{+}$.

The increase of void growth can be clearly observed in Fig. 16. Regarding the onset of void coalescence, it is at $7.21 \%$ for $T_{0}, 15.89 \%$ for $T_{-}$, and $2.67 \%$ for $T_{+}$. After this onset, coalescence propagates faster at high triaxiality, which is in agreement with the literature. An interesting result here is that due to higher necking in the horizontal direction at lower triaxiality, the void shape is different. The void shape at the onset of coalescence is obviously also influenced by the fact that this onset is delayed at lower stress triaxiality. Therefore, void coalescence propagation is also influenced in shape, and the propagation path may be different depending on triaxiality. This is illustrated in Fig. 17. 


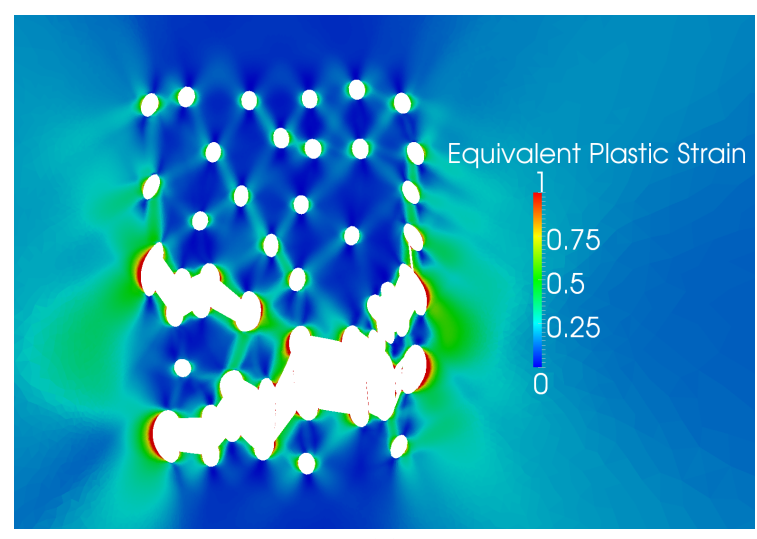

(a)

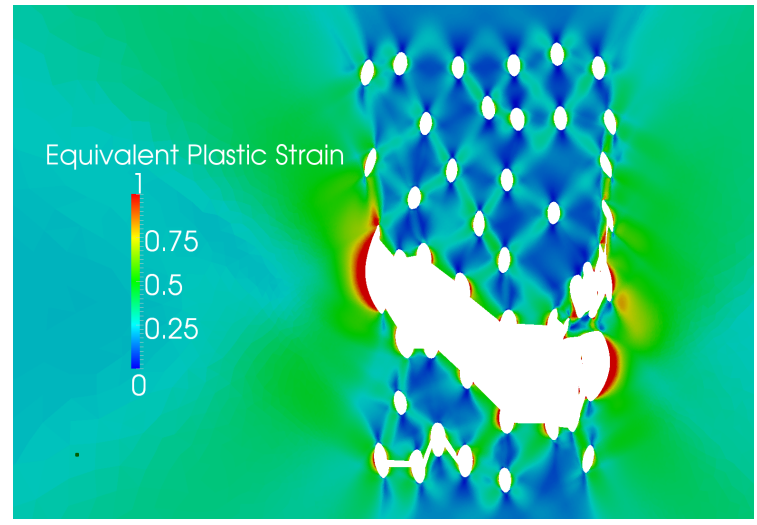

(b)

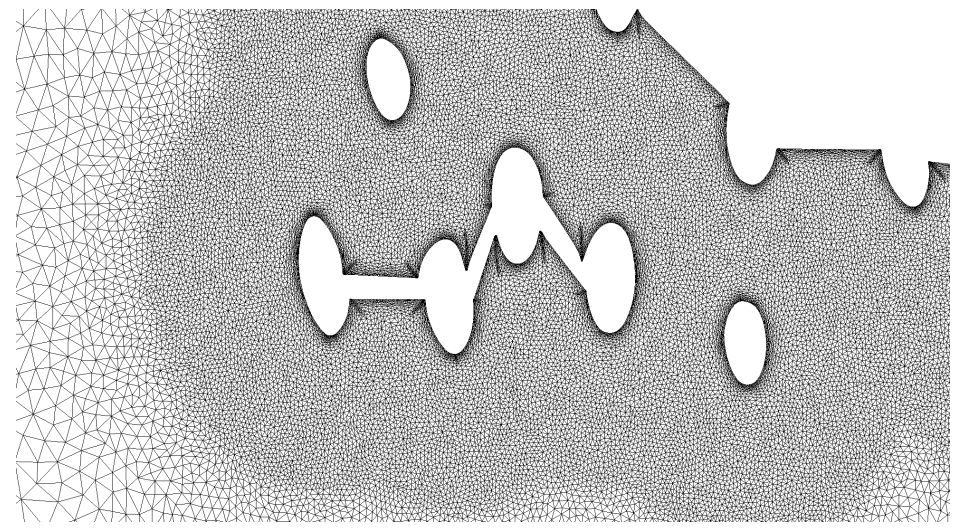

(c)

Figure 17: Comparison between the void shape at $12.5 \%$ of domain elongation for high stress triaxiality (a), and the void shape at $25 \%$ of domain elongation for uniaxial tension $(\mathrm{b}, \mathrm{c})$.

These results reveal the importance of finite element analysis not only until the onset of void coalescence, but also after. Another conclusion that can be raised with these results, and more particularly regarding the study of random void arrangements, is that it is important to bring finite element analysis closer to real microstructures and real conditions. If these results and this work are first steps towards such end, then the following step would obviously be 3D computations. For this purpose, some measures of computational costs are summarized in Tab. 6.

\begin{tabular}{l|ccc} 
Simulation & Reference case (two voids at 45 $)$ & Random Ar. 0 & Random Ar. 4 \\
\hline Number of elements & $50000-150000$ & $150000-360000$ & $150000-350000$ \\
Number of cores & 16 & 64 & 64 \\
Computation time (h) & 10 & 59 & 37 \\
Mechanical resolution time (\%) & 64 & 37 & 61 \\
Coalescence criterion time (\%) & 1 & 45 & 12 \\
Remeshing time (\%) & 1 & 1 & 1 \\
LS reinitialization time (\%) & 1 & 14 & 4 \\
Output/post-process time (\%) & 33 & 3 & 22
\end{tabular}

Table 6: Mesh statistics and computation times for some simulations.

The simulations were performed on a cluster of HP Proliant nodes with a $2.3 \mathrm{GHz}$ AMD Opteron 16-core processor and 32 GB RAM. Note that since data outputting/post-processing was performed systematically 
here (at each iteration) for the purpose of numerical investigation, the cost of these operations is overestimated. It is clear that remeshing and LS reinitialization are not costly at all, at least in 2D. Some further work on remeshing could be investigated, for example to increase even more mesh quality and reduce mechanical resolution time, which is the largest contribution to total computation time. Regarding the coalescence criterion, its implementation could be optimized, for example by applying the space partitioning technique used in [34] for LS reinitialization. Finally, increasing the time step could reduce the total computation time without having too much impact on accuracy, as shown in section 4.1.4. Further study of the issue encountered in section 4.1 .3 could also enable important computation time reduction through mesh coarsening. Indeed, the mesh illustrated in $17(\mathrm{c})$ seems too fine and not reasonable for $3 \mathrm{D}$ computations.

\section{Conclusion}

In this paper, a new body-fitted meshing and remeshing methodology has been presented for immersed volume applications. While the present paper focuses on ductile fracture and void coalescence in a two phase material (matrix and void), the proposed remeshing method has a wide range of potential applications such as the modeling of fluid/structure interactions. Another application is obviously the simulation of void nucleation, where matrix/inclusion interfaces would be meshed accurately initially to predict interface debonding or inclusion fracture, and then remeshed after the occurrence of such events. The method itself requires some improvements regarding volume conservation in order to prevent any mesh dependency of the solution. Nevertheless, the results have proved the ability of the new tool, Fitz, to capture new interfaces on-the-fly during the simulation and to maintain a good simplex quality at high deformations, especially close to interfaces.

Regarding void coalescence, this immersed volume framework has been the basis for a complete mechanical modeling. This modeling includes nonlinear material behavior and also a damage and void coalescence model. After numerical validation, the framework has been applied to the analysis of two main topics: void arrangement and stress state effects. The definition of the RVE and the boundary conditions were particular, and for future works it seems important to bring the simulation set-up closer to what is done in the rest of the literature. Yet, interesting results were obtained and proved an important influence of relative void positioning and clustering on the overall ductility of a microstructure. Regarding stress state effects, it was shown that increasing the triaxiality accelerates void growth and void coalescence, and changes its propagation path.

The proposed method also opens new opportunities, as it can be applied to random and hence real microstructures. An interesting study would be to compare simulation results with experiments by retrieving accurate boundary conditions using DVC and applying them to the real microstructures. Before considering such simulations, it is essential to verify the applicability of the present framework to 3D situations. Though the presented numerical methods and models do not theoretically present any such limitation, quantification of computational costs seems unavoidable for practical use.

\section{Acknowledgements}

The support of the Carnot M.I.N.E.S institute is gratefully acknowledged.

\section{References}

[1] T.-S. Cao, A. Gaillac, P. Montmitonnet, P.-O. Bouchard, Identification methodology and comparison of phenomenological ductile damage models via hybrid numerical-experimental analysis of fracture experiments conducted on a zirconium alloy, International Journal of Solids and Structures 50 (24) (2013) 3984-3999. doi:10.1016/j.ijsolstr.2013.08.011.

[2] T.-S. Cao, C. Bobadilla, P. Montmitonnet, P.-O. Bouchard, A comparative study of three ductile damage approaches for fracture prediction in cold forming processes, Journal of Materials Processing Technology 216 (2015) 385-404. doi:10.1016/j.jmatprotec.2014.10.009.

[3] T.-S. Cao, P. Montmitonnet, P.-O. Bouchard, A detailed description of the Gurson-Tvergaard-Needleman model within a mixed velocity-pressure finite element formulation, International Journal for Numerical Methods in Engineering 96 (9) (2013) 561-583. doi:10.1002/nme.4571. 
[4] T.-S. Cao, E. Maire, C. Verdu, C. Bobadilla, P. Lasne, P. Montmitonnet, P.-O. Bouchard, Characterization of ductile damage for a high carbon steel using 3D X-ray micro-tomography and mechanical tests - Application to the identification of a shear modified GTN model, Computational Materials Science 84 (2014) 175-187. doi:10.1016/j.commatsci.2013.12.006.

[5] J. Gammage, D. Wilkinson, Y. Brechet, D. Embury, A model for damage coalescence in heterogeneous multi-phase materials, Acta Materialia 52 (18) (2004) 5255-5263. doi:10.1016/j.actamat.2004.07.009.

[6] D. Lassance, F. Scheyvaerts, T. Pardoen, Growth and coalescence of penny-shaped voids in metallic alloys, Engineering Fracture Mechanics 73 (8) (2006) 1009-1034. doi:10.1016/j.engfracmech.2005.12.004.

[7] D. Lassance, D. Fabregue, F. Delannay, T. Pardoen, Micromechanics of room and high temperature fracture in 6xxx Al alloys, Progress in Materials Science 52 (1) (2007) 62-129. doi:10.1016/j.pmatsci.2006.06.001.

[8] D. Fabrègue, T. Pardoen, A constitutive model for elastoplastic solids containing primary and secondary voids, Journal of the Mechanics and Physics of Solids 56 (3) (2008) 719-741. doi:10.1016/j.jmps.2007.07.008.

[9] F. Scheyvaerts, T. Pardoen, P. Onck, A New Model for Void Coalescence by Internal Necking, International Journal of Damage Mechanics 19 (1) (2009) 95-126. doi:10.1177/1056789508101918.

[10] A. A. Benzerga, J. B. Leblond, Ductile Fracture by Void Growth to Coalescence, Advances in Applied Mechanics 44 (2010) 169-305. doi:10.1016/S0065-2156(10)44003-X.

[11] K. L. Nielsen, V. Tvergaard, Failure by void coalescence in metallic materials containing primary and secondary voids subject to intense shearing, International Journal of Solids and Structures 48 (9) (2011) $1255-1267$. doi:10.1016/j.ijsolstr.2011.01.008.

[12] F. Scheyvaerts, P. Onck, C. Tekoglu, T. Pardoen, The growth and coalescence of ellipsoidal voids in plane strain under combined shear and tension, Journal of the Mechanics and Physics of Solids 59 (2) (2011) $373-397$. doi:10.1016/j.jmps.2010.10.003.

[13] C. Tekoglu, J.-B. Leblond, T. Pardoen, A criterion for the onset of void coalescence under combined tension and shear, Journal of the Mechanics and Physics of Solids 60 (7) (2012) 1363-1381. doi:10.1016/j.jmps.2012.02.006.

[14] E. Roux, M. Bernacki, P. Bouchard, A level-set and anisotropic adaptive remeshing strategy for the modeling of void growth under large plastic strain, Computational Materials Science 68 (2013) 32-46. doi:10.1016/j.commatsci.2012.10.004.

[15] E. Roux, M. Shakoor, M. Bernacki, P.-O. Bouchard, A new finite element approach for modelling ductile damage void nucleation and growth - analysis of loading path effect on damage mechanisms, Modelling and Simulation in Materials Science and Engineering 22 (7) (2014) 075001. doi:10.1088/0965-0393/22/7/075001.

[16] T. Coupez, H. Digonnet, R. Ducloux, Parallel meshing and remeshing, Applied Mathematical Modelling 25 (2) (2000) 153-175. doi:10.1016/S0307-904X(00)00045-7.

[17] C. Gruau, T. Coupez, 3D tetrahedral, unstructured and anisotropic mesh generation with adaptation to natural and multidomain metric, Computer Methods in Applied Mechanics and Engineering 194 (48-49) (2005) 4951-4976. doi:10.1016/j.cma.2004.11.020.

[18] P. Laure, G. Baume, O. Basset, S. Luisa, T. Coupez, Numerical methods for solid particles in particulate flow simulations, Revue européenne de mécanique numérique 16 (3-4) (2007) 365-383. doi:10.3166/remn.16.365-383.

[19] Z. Sun, R. Logé, M. Bernacki, 3D finite element model of semi-solid permeability in an equiaxed granular structure, Computational Materials Science 49 (1) (2010) 158-170. doi:10.1016/j.commatsci.2010.04.042.

[20] E. Hachem, S. Feghali, R. Codina, T. Coupez, Immersed stress method for fluid-structure interaction using anisotropic mesh adaptation, International Journal for Numerical Methods in Engineering 94 (9) (2013) 805-825. doi: $10.1002 /$ nme.4481.

[21] L. Silva, R. Valette, P. Laure, T. Coupez, A new three-dimensional mixed finite element for direct numerical simulation of compressible viscoelastic flows with moving free surfaces, International Journal of Material Forming 5 (1) (2011) 55-72. doi:10.1007/s12289-011-1030-2.

[22] H. Resk, L. Delannay, M. Bernacki, T. Coupez, R. Logé, Adaptive mesh refinement and automatic remeshing in crystal plasticity finite element simulations, Modelling and Simulation in Materials Science and Engineering 17 (7) (2009) 075012. doi:10.1088/0965-0393/17/7/075012.

[23] G. Compère, J. F. Remacle, E. Marchandise, Transient Mesh Adaptivity with Large Rigid-Body Displacements, in: R. V. Garimella (Ed.), Proceedings of the 17th International Meshing Roundtable, Springer Berlin Heidelberg, Berlin, Heidelberg, 2008, pp. 213-230. doi:10.1007/978-3-540-87921-3_13.

[24] G. Compere, J. F. Remacle, J. Jansson, J. Hoffman, A mesh adaptation framework for dealing with large deforming meshes, International Journal for Numerical Methods in Engineering 82 (November 2009) (2009) 843-867. doi:10.1002/nme.2788.

[25] J. Hoffman, J. Jansson, R. Vilela de Abreu, N. C. Degirmenci, N. Jansson, K. Müller, M. Nazarov, J. H. Spühler, Unicorn: Parallel adaptive finite element simulation of turbulent flow and fluid-structure interaction for deforming domains and complex geometry, Computers \& Fluids 80 (2013) 310-319. doi:10.1016/j.compfluid.2012.02.003.

[26] M. Bernacki, H. Resk, T. Coupez, R. E. Logé, Finite element model of primary recrystallization in polycrystalline aggregates using a level set framework, Modelling and Simulation in Materials Science and Engineering 17 (6) (2009) 064006. doi:10.1088/0965-0393/17/6/064006.

[27] J. F. Remacle, M. S. Shephard, An algorithm oriented mesh database, International Journal for Numerical Methods in Engineering 58 (2) (2003) 349-374. doi:10.1002/nme.774.

[28] K. J. Fidkowski, D. L. Darmofal, A triangular cut-cell adaptive method for high-order discretizations of the compressible Navier-Stokes equations, Journal of Computational Physics 225 (2) (2007) 1653-1672. doi:10.1016/j.jcp.2007.02.007.

[29] D.-L. Quan, T. Toulorge, E. Marchandise, J.-F. Remacle, G. Bricteux, Anisotropic mesh adaptation with optimal convergence for finite elements using embedded geometries, Computer Methods in Applied Mechanics and Engineering 268 (2014) 65-81. doi:10.1016/j.cma.2013.09.007.

[30] Y. Belhamadia, A. Fortin, E. Chamberland, Anisotropic mesh adaptation for the solution of the Stefan problem, Journal 
of Computational Physics 194 (1) (2004) 233-255. doi:10.1016/j.jcp.2003.09.008.

[31] C. Gruau, Génération de métriques pour adaptation anisotrope de maillages, applications à la mise en forme des matériaux, Phd, Ecole Nationale Supérieure des Mines de Paris (2004).

[32] D. Boffi, F. Brezzi, L. F. Demkowicz, R. G. Durán, R. S. Falk, M. Fortin, Mixed Finite Elements, Compatibility Conditions, and Applications, Vol. 1939 of Lecture Notes in Mathematics, Springer Berlin Heidelberg, Berlin, Heidelberg, 2008. doi:10.1007/978-3-540-78319-0.

[33] R. H. Wagoner, J. L. Chenot, Metal Forming Analysis, Cambridge University Press, 2001.

[34] M. Shakoor, B. Scholtes, P.-O. Bouchard, M. Bernacki, An efficient and parallel level set reinitialization method - Application to micromechanics and microstructural evolutions, Applied Mathematical Modelling Submitted. doi:10.1016/j.apm.2015.03.014.

[35] L. Babout, E. Maire, J. Y. Buffière, R. Fougeres, Characterization by X-ray computed tomography of decohesion, porosity growth and coalescence in model metal matrix composites, Acta Materialia 49 (11) (2001) 2055-2063.

[36] J. Bandstra, D. Koss, A. Geltmacher, P. Matic, R. Everett, Modeling void coalescence during ductile fracture of a steel, Materials Science and Engineering: A 366 (2) (2004) 269-281. doi:10.1016/j.msea.2003.08.018.

[37] T. F. Morgeneyer, T. Taillandier-Thomas, L. Helfen, T. Baumbach, I. Sinclair, S. Roux, F. Hild, In situ 3-D observation of early strain localization during failure of thin Al alloy (2198) sheet, Acta Materialia 69 (2014) 78-91. doi:10.1016/j.actamat.2014.01.033.

[38] T. F. Morgeneyer, L. Helfen, H. Mubarak, F. Hild, 3D Digital Volume Correlation of Synchrotron Radiation Laminography Images of Ductile Crack Initiation: An Initial Feasibility Study, Experimental Mechanics 53 (4) (2012) 543-556. doi:10.1007/s11340-012-9660-y.

[39] A. Weck, T. H. R. Crawford, A. Borowiec, D. S. Wilkinson, J. S. Preston, Femtosecond laser-based fabrication of a new model material to study fracture, Applied Physics A 86 (1) (2006) 55-61. doi:10.1007/s00339-006-3730-x.

[40] A. Weck, D. Wilkinson, Experimental investigation of void coalescence in metallic sheets containing laser drilled holes, Acta Materialia 56 (8) (2008) 1774-1784. doi:10.1016/j.actamat.2007.12.035.

[41] A. Weck, D. S. Wilkinson, E. Maire, H. Toda, Visualization by X-ray tomography of void growth and coalescence leading to fracture in model materials, Acta Materialia 56 (12) (2008) 2919-2928. doi:10.1016/j.actamat.2008.02.027.

[42] R. Peerlings, L. Poh, M. Geers, An implicit gradient plasticity-damage theory for predicting size effects in hardening and softening, Engineering Fracture Mechanics 95 (2012) 2-12. doi:10.1016/j.engfracmech.2011.12.016.

[43] R. El khaoulani, Prédiction fiable de l'endommagement ductile par la méthode des éléments finis mixtes : endommagement non local et adaptation de maillage, Ph.D. thesis, Ecole Nationale Supérieure des Mines de Paris (2010).

[44] Y. Alinaghian, M. Asadi, A. Weck, Effect of pre-strain and work hardening rate on void growth and coalescence in AA5052, International Journal of Plasticity 53 (2014) 193-205. doi:10.1016/j.ijplas.2013.08.007.

[45] A. Hosokawa, D. S. Wilkinson, J. Kang, E. Maire, Effect of triaxiality on void growth and coalescence in model materials investigated by X-ray tomography, Acta Materialia 60 (6-7) (2012) 2829-2839. doi:10.1016/j.actamat.2012.01.048. 